\title{
On Optimization of Manufacturing of Bipolar Heterotransistors Framework Circuit of a High-voltage Element or to Increase Their Integration Rate: On Influence Mismatch-induced Stress
}

\author{
E. L. Pankratov ${ }^{1,2}$ \\ ${ }^{1}$ Nizhny Novgorod State University, Russia \\ ${ }^{2}$ Nizhny Novgorod State Technical University, Russia
}

Copyright $\bigcirc 2019$ by authors, all rights reserved. Authors agree that this article remains permanently open access under the terms of the Creative Commons Attribution License 4.0 International License

\begin{abstract}
In this paper, we introduce an approach to decrease dimensions of bipolar heterotransistors framework a circuit of a voltage divider biasing common emitter amplifier. Framework of the approach, we consider manufacturing of the divider in heterostructure with specific configuration. Several specific areas of the heterostructure should be doped by diffusion or by ion implantation. After this doping, dopant and/or radiation defects should be annealed by using optimized scheme. We also consider an approach to decrease value of mismatch-induced stress in the considered heterostructure. To make prognosis of technological process and obtain recommendations to optimize the process, we introduce an analytical approach to analyze mass and heat transport in heterostructures with account mismatch-induced stress.
\end{abstract}

Keywords Voltage Divider Biasing Common Emitter Amplifier, Increasing Integration Rate of Bipolar Transistors, Optimization of Manufacturing

\section{Introduction}

In the present time, several actual problems of solid state electronics (such as increasing of performance, reliability and density of elements of integrated circuits: diodes, field-effect and bipolar transistors) are intensively solving [1-6]. To increase the performance of these devices, it is attracted an interest determination of materials with higher values of charge carriers mobility [7-10]. One way to decrease dimensions of elements of integrated circuits is manufacturing them in thin film heterostructures $[3-5,11]$. In this case, it is possible to use inhomogeneity of heterostructure to improve properties of considered devices. However, it is necessary to optimize doping of electronic materials $[12,13]$ and development of epitaxial technology to improve considered materials (including analysis of mismatch induced stress) [14-16]. An alternative approach to using heterostructures is using laser or microwave types of annealing [17-19].

Framework of the paper, we introduce an approach to manufacture a bipolar transistor. The approach gives a possibility to decrease dimensions of the transistor framework a voltage divider biasing common emitter amplifier. We also consider possibility to decrease mismatch-induced stress to decrease quantity of defects, generated due to the stress. In this paper, we consider a heterostructure, which consists of a substrate and an epitaxial layer (see Fig. 1). We also consider a buffer layer between the substrate and the epitaxial layer. The epitaxial layer includes into itself several sections, which were manufactured by using other materials. These sections have been doped by diffusion or ion implantation to manufacture the required types of conductivity ( $p$ or $n$ ). These areas became sources, drains and gates (see Fig. 1). After this doping it is required annealing of dopant and/or radiation defects. Main aim of the present paper is analysis of redistribution of dopant and radiation defects to determine conditions, which correspond to decreasing of elements of the considered divider and at the same time to increase their density. At the same time we consider a possibility to decrease mismatch-induced stress. 


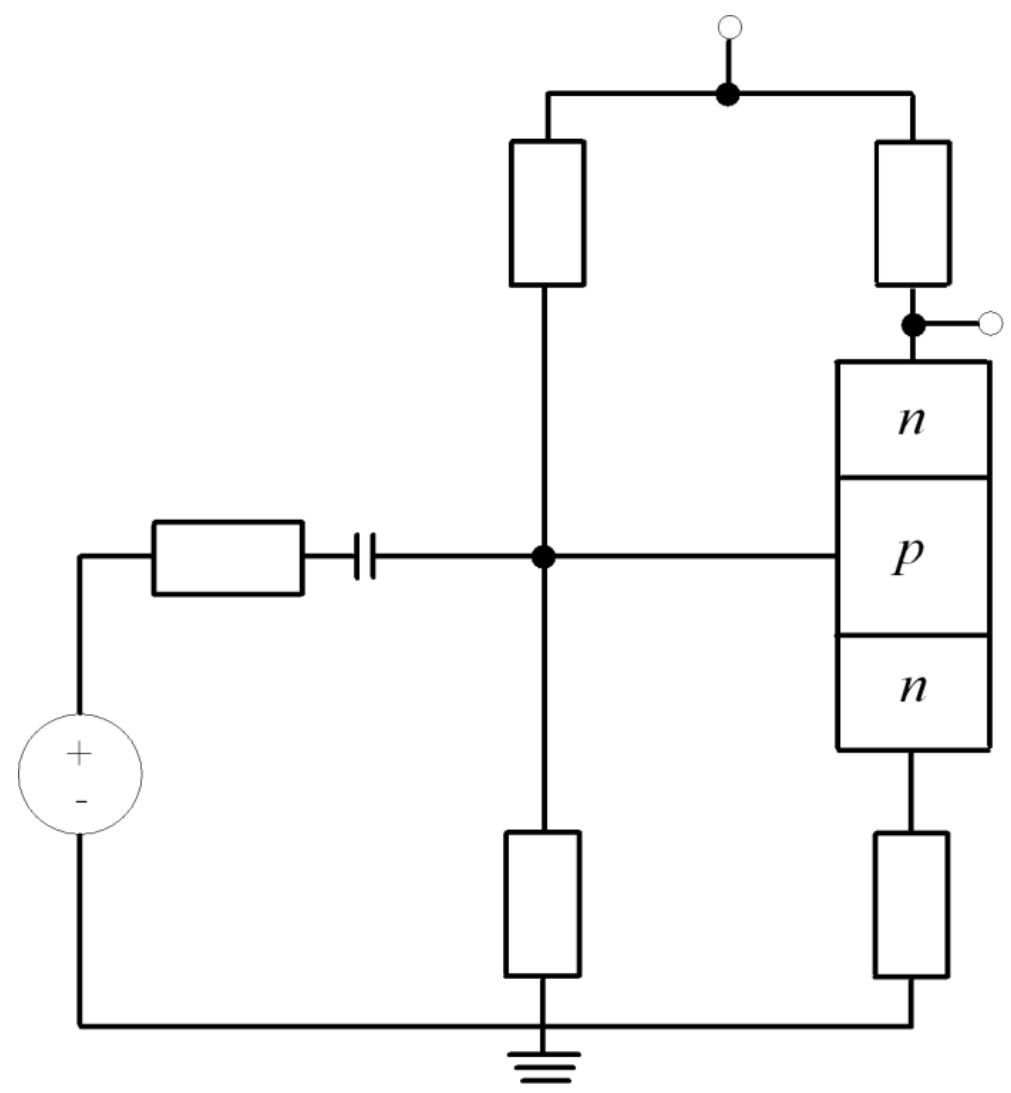

Figure 1a. Structure of the considered voltage divider [13]

\begin{tabular}{|c|}
\hline Epitaxial layer \\
\hline Buffer layer \\
\hline Substrate \\
\\
\hline
\end{tabular}

Figure 1 $\boldsymbol{b}$. Heterostructure with a substrate, epitaxial layers and buffer layer (view from side)

\section{Method of Solution}

To solve our aim, we determine and analyze spatio-temporal distribution of concentration of dopant in the considered heterostructure. We determine the distribution by solving of the second Fick's law in the following form [1, 20-24]

$$
\begin{aligned}
\frac{\partial C(x, y, z, t)}{\partial t}= & \frac{\partial}{\partial x}\left[D \frac{\partial C(x, y, z, t)}{\partial x}\right]+\frac{\partial}{\partial y}\left[D \frac{\partial C(x, y, z, t)}{\partial y}\right]+\frac{\partial}{\partial z}\left[D \frac{\partial C(x, y, z, t)}{\partial z}\right]+ \\
& +\Omega \frac{\partial}{\partial x}\left[\frac{D_{S}}{k T} \nabla_{S} \mu_{1}(x, y, z, t) \int_{0}^{L_{z}} C(x, y, W, t) d W\right]+
\end{aligned}
$$




$$
+\Omega \frac{\partial}{\partial y}\left[\frac{D_{s}}{k T} \nabla_{s} \mu_{1}(x, y, z, t) \int_{0}^{L_{z}} C(x, y, W, t) d W\right]
$$

Here the first, the second and the third terms in right side of Eq. (1) describe thermal diffusion of dopant. The forth and the fifth terms of this equation describe transport of dopant under influence of mismatch-induced stress. Boundary (correspond to absents of dopant flow through external boundary of considered heterostructure) and initial conditions for Eq.(1) are

$$
\begin{gathered}
\left.\frac{\partial C(x, y, z, t)}{\partial x}\right|_{x=0}=0,\left.\frac{\partial C(x, y, z, t)}{\partial x}\right|_{x=L_{x}}=0,\left.\frac{\partial C(x, y, z, t)}{\partial y}\right|_{y=0}=0, C(\mathrm{x}, \mathrm{y}, \mathrm{z}, 0)=\mathrm{f}_{\mathrm{C}}(\mathrm{x}, \mathrm{y}, \mathrm{z}), \\
\left.\frac{\partial C(x, y, z, t)}{\partial y}\right|_{x=L_{y}}=\left.0 \frac{\partial C(x, y, z, t)}{\partial z}\right|_{z=0}=\left.0 \frac{\partial C(x, y, z, t)}{\partial z}\right|_{x=L_{z}}=0 .
\end{gathered}
$$

Function $C(x, y, z, t)$ describes the spatio-temporal distribution of concentration of dopant; $\Omega$ is the atomic volume of dopant; $\nabla_{s}$ is the symbol of surficial gradient; $\int_{0}^{L_{z}} C(x, y, z, t) d z$ is the surficial concentration of dopant on interface between layers of heterostructure (in this situation we assume, that Z-axis is perpendicular to interface between layers of heterostructure); $\mu_{1}(x, y, z, t)$ is the chemical potential due to the presence of mismatch-induced stress; $D$ and $D_{S}$ are the coefficients of volumetric and surficial diffusions. Values of dopant diffusions coefficients depends on properties of materials of heterostructure, speed of heating and cooling of materials during annealing and spatio-temporal distribution of concentration of dopant. Dependences of dopant diffusions coefficients on parameters could be approximated by the following relations [22-24]

$$
\begin{aligned}
& D_{C}=D_{L}(x, y, z, T)\left[1+\xi \frac{C^{\gamma}(x, y, z, t)}{P^{\gamma}(x, y, z, T)}\right]\left[1+\varsigma_{1} \frac{V(x, y, z, t)}{V^{*}}+\varsigma_{2} \frac{V^{2}(x, y, z, t)}{\left(V^{*}\right)^{2}}\right] \\
& D_{S}=D_{S L}(x, y, z, T)\left[1+\xi_{S} \frac{C^{\gamma}(x, y, z, t)}{P^{\gamma}(x, y, z, T)}\right]\left[1+\varsigma_{1} \frac{V(x, y, z, t)}{V^{*}}+\varsigma_{2} \frac{V^{2}(x, y, z, t)}{\left(V^{*}\right)^{2}}\right] .
\end{aligned}
$$

Here the first multipliers in the right side of Eqs. (2) describe coefficient of linear diffusion. Nonlinearity of diffusion (in the high doped case [22]) was taken into account by the second multipliers in the Eqs. (2). The third multipliers of Eqs. (2) give a possibility radiation damage of materials of heterostructure [23,24]. Functions $D_{L}(x, y, z, T)$ and $D_{L S}(x, y, z, T)$ in Eqs. (2) describe the spatial (due to accounting layers of heterostruicture) and temperature (due to Arrhenius law) dependences of dopant diffusion coefficients; $T$ is the temperature of annealing; $P(x, y, z, T)$ is the limit of solubility of dopant; parameter $\gamma$ depends on properties of materials and could be integer in the following interval $\gamma \in[1,3][22] ; V$ $(x, y, z, t)$ is the spatio-temporal distribution of concentration of radiation vacancies; $V^{*}$ is the equilibrium distribution of vacancies. Concentrational dependence of dopant diffusion coefficient has been described in details in [22]. Spatio-temporal distributions of concentration of point radiation defects have been determined by solving the following system of equations [20, 23, 24]

$$
\begin{aligned}
& \frac{\partial I(x, y, z, t)}{\partial t}=\frac{\partial}{\partial x}\left[D_{I}(x, y, z, T) \frac{\partial I(x, y, z, t)}{\partial x}\right]+\frac{\partial}{\partial y}\left[D_{I}(x, y, z, T) \frac{\partial I(x, y, z, t)}{\partial y}\right]+ \\
& +\frac{\partial}{\partial z}\left[D_{I}(x, y, z, T) \frac{\partial I(x, y, z, t)}{\partial z}\right]-k_{I, I}(x, y, z, T) I^{2}(x, y, z, t)-k_{I, V}(x, y, z, T) \times
\end{aligned}
$$




$$
\begin{gathered}
\times I(x, y, z, t) V(x, y, z, t)+\Omega \frac{\partial}{\partial x}\left[\frac{D_{I S}}{k T} \nabla_{S} \mu(x, y, z, t) \int_{0}^{L_{z}} I(x, y, W, t) d W\right]+ \\
+\Omega \frac{\partial}{\partial y}\left[\frac{D_{I S}}{k T} \nabla_{s} \mu(x, y, z, t) \int_{0}^{L_{z}} I(x, y, W, t) d W\right] \\
\frac{\partial V(x, y, z, t)}{\partial t}=\frac{\partial}{\partial x}\left[D_{V}(x, y, z, T) \frac{\partial V(x, y, z, t)}{\partial x}\right]+\frac{\partial}{\partial y}\left[D_{V}(x, y, z, T) \frac{\partial V(x, y, z, t)}{\partial y}\right]+ \\
+\frac{\partial}{\partial z}\left[D D_{V}(x, y, z, T) \frac{\partial V(x, y, z, t)}{\partial z}\right]-k_{V, V}(x, y, z, T) V^{2}(x, y, z, t)-k_{I, V}(x, y, z, T) \times \\
\times I(x, y, z, t) V(x, y, z, t)+\Omega \frac{\partial}{\partial x}\left[\frac{D_{V S}}{k T} \nabla_{S} \mu(x, y, z, t) \int_{0}^{L_{z}} V(x, y, W, t) d W\right]+ \\
+\Omega \frac{\partial}{\partial y}\left[\frac{D_{V S}}{k T} \nabla_{S} \mu(x, y, z, t) \int_{0}^{L_{z}} V(x, y, W, t) d W\right]
\end{gathered}
$$

with boundary and initial conditions

$$
\begin{aligned}
& \left.\frac{\partial I(x, y, z, t)}{\partial x}\right|_{x=0}=\left.0 \frac{\partial I(x, y, z, t)}{\partial x}\right|_{x=L_{x}}=\left.0 \frac{\partial I(x, y, z, t)}{\partial y}\right|_{y=0}=\left.0 \frac{\partial I(x, y, z, t)}{\partial y}\right|_{y=L_{y}}=0 \\
& \left.\frac{\partial I(x, y, z, t)}{\partial z}\right|_{z=0}=\left.0 \frac{\partial I(x, y, z, t)}{\partial z}\right|_{z=L_{z}}=\left.0 \frac{\partial V(x, y, z, t)}{\partial x}\right|_{x=0}=\left.0 \frac{\partial V(x, y, z, t)}{\partial x}\right|_{x=L_{x}}=0, \\
& \left.\frac{\partial V(x, y, z, t)}{\partial y}\right|_{y=0}=\left.0 \frac{\partial V(x, y, z, t)}{\partial y}\right|_{y=L_{y}}=0 \frac{\partial V(x, y, z, t)}{\partial z}=\left.0 \frac{\partial V(x, y, z, t)}{\partial z}\right|_{z=0}=0, \\
& \mathrm{I}(\mathrm{x}, \mathrm{y}, \mathrm{z}, 0)=\mathrm{f}_{\mathrm{I}}(\mathrm{x}, \mathrm{y}, \mathrm{z}), \mathrm{V}(\mathrm{x}, \mathrm{y}, \mathrm{z}, 0)=\mathrm{f}_{\mathrm{V}}(\mathrm{x}, \mathrm{y}, \mathrm{z}) .
\end{aligned},
$$

Here $I(x, y, z, t)$ is the spatio-temporal distribution of concentration of radiation interstitials; $I^{*}$ is the equilibrium distribution of interstitials; $D_{I}(x, y, z, T), D_{V}(x, y, z, T), D_{I S}(x, y, z, T), D_{V S}(x, y, z, T)$ are the coefficients of volumetric and surficial diffusions of interstitials and vacancies, respectively; $k_{I, V}(x, y, z, T), k_{I, I}(x, y, z, T)$ and $k_{V, V}(x, y, z, T)$ are the parameters of recombination of point radiation defects and generation of their complexes. The first, the second and the third terms in right side of Eqs.(3) describe thermal diffusion of point defects. The forth terms of Eqs.(3) describe generation of simple complexes of point defects (divacancies and diinterstitials; see, for example, [24] and appropriate references in this book). The fives terms of Eqs.(3) describe recombination of point defects. The sixth and the seventh terms of Eqs.(3) describe transport of point defects under influence of mismatch-induced stress. All boundary conditions correspond to absent of flow of defects through external boundary of the considered heterostructure.

Spatio-temporal distributions of divacancies $\Phi_{V}(x, y, z, t)$ and diinterstitials $\Phi_{I}(x, y, z, t)$ could be determined by solving the following system of equations $[20,23,24]$

$$
\frac{\partial \Phi_{I}(x, y, z, t)}{\partial t}=\frac{\partial}{\partial x}\left[D_{\Phi_{I}}(x, y, z, T) \frac{\partial \Phi_{I}(x, y, z, t)}{\partial x}\right]+\frac{\partial}{\partial y}\left[D_{\Phi_{I}}(x, y, z, T) \frac{\partial \Phi_{I}(x, y, z, t)}{\partial y}\right]+
$$




$$
\begin{aligned}
& +\frac{\partial}{\partial z}\left[D_{\Phi_{I}}(x, y, z, T) \frac{\partial \Phi_{I}(x, y, z, t)}{\partial z}\right]+\Omega \frac{\partial}{\partial x}\left[\frac{D_{\Phi_{I} S}}{k T} \nabla_{S} \mu_{1}(x, y, z, t) \int_{0}^{L_{z}} \Phi_{I}(x, y, W, t) d W\right]+ \\
& +\Omega \frac{\partial}{\partial y}\left[\frac{D_{\Phi_{I} S}}{k T} \nabla_{S} \mu_{1}(x, y, z, t) \int_{0}^{L_{z}} \Phi_{I}(x, y, W, t) d W\right]+k_{I, I}(x, y, z, T) I^{2}(x, y, z, t)+ \\
& +k_{I}(x, y, z, T) I(x, y, z, t) \\
& \frac{\partial \Phi_{V}(x, y, z, t)}{\partial t}=\frac{\partial}{\partial x}\left[D_{\Phi_{V}}(x, y, z, T) \frac{\partial \Phi_{V}(x, y, z, t)}{\partial x}\right]+\frac{\partial}{\partial y}\left[D_{\Phi_{V}}(x, y, z, T) \frac{\partial \Phi_{V}(x, y, z, t)}{\partial y}\right]+ \\
& +\frac{\partial}{\partial z}\left[D_{\Phi_{V}}(x, y, z, T) \frac{\partial \Phi_{V}(x, y, z, t)}{\partial z}\right]+\Omega \frac{\partial}{\partial x}\left[\frac{D_{\Phi_{V} S}}{k T} \nabla_{S} \mu_{1}(x, y, z, t) \int_{0}^{L_{z}} \Phi_{V}(x, y, W, t) d W\right]+ \\
& +\Omega \frac{\partial}{\partial y}\left[\frac{D_{\Phi_{V} S}}{k T} \nabla_{S} \mu_{1}(x, y, z, t) \int_{0}^{L_{z}} \Phi_{V}(x, y, W, t) d W\right]+k_{V, V}(x, y, z, T) V^{2}(x, y, z, t)+ \\
& +k_{V}(x, y, z, T) V(x, y, z, t)
\end{aligned}
$$

with boundary and initial conditions

$$
\begin{aligned}
& \left.\frac{\partial \Phi_{I}(x, y, z, t)}{\partial x}\right|_{x=0}=\left.0 \frac{\partial \Phi_{I}(x, y, z, t)}{\partial x}\right|_{x=L_{x}}=\left.0 \frac{\partial \Phi_{I}(x, y, z, t)}{\partial y}\right|_{y=0}=0 \\
& \left.\frac{\partial \Phi_{I}(x, y, z, t)}{\partial y}\right|_{y=L_{y}}=\left.0 \frac{\partial \Phi_{I}(x, y, z, t)}{\partial z}\right|_{z=0}=\left.0 \frac{\partial \Phi_{I}(x, y, z, t)}{\partial z}\right|_{z=L_{z}}=0 \\
& \left.\frac{\partial \Phi_{V}(x, y, z, t)}{\partial x}\right|_{x=0}=0,\left.\frac{\partial \Phi_{V}(x, y, z, t)}{\partial x}\right|_{x=L_{x}}=0,\left.\frac{\partial \Phi_{V}(x, y, z, t)}{\partial y}\right|_{y=0}=0, \\
& \left.\frac{\partial \Phi_{V}(x, y, z, t)}{\partial y}\right|_{y=L_{y}}=\left.0 \quad \frac{\partial \Phi_{V}(x, y, z, t)}{\partial z}\right|_{z=0}=\left.0 \quad \frac{\partial \Phi_{V}(x, y, z, t)}{\partial z}\right|_{z=L_{z}}=0
\end{aligned}
$$

Here $D_{\phi I}(x, y, z, T), D_{\Phi \vee}(x, y, z, T), D_{\Phi I S}(x, y, z, T)$ and $D_{\Phi V S}(x, y, z, T)$ are the coefficients of volumetric and surficial diffusions of simplest complexes of radiation defects; $k_{I}(x, y, z, T)$ and $k_{V}(x, y, z, T)$ are the parameters of decay of complexes of radiation defects. The first, the second and the third terms in right side of Eqs.(5) describe thermal diffusion of simplest complexes of point defects. The forth and the fives terms of Eqs.(5) describe transport of divacancies and diinterstitials under influence of mismatch-induced stress. The sixth terms of Eqs.(5) describe generation of divacancies and diinterstitials. The seventh terms of Eqs.(5) describe decay of complexes of point radiation defects. All boundary conditions correspond to absent of flow of defects through external boundary of the considered heterostructure.

Chemical potential $\mu_{1}$ in Eq.(1) could be determine by the following relation [20]

$$
\mu_{1}=\mathrm{E}(\mathrm{z}) \Omega \sigma_{\mathrm{ij}}\left[\mathrm{u}_{\mathrm{ij}}(\mathrm{x}, \mathrm{y}, \mathrm{z}, \mathrm{t})+\mathrm{u}_{\mathrm{ji}}(\mathrm{x}, \mathrm{y}, \mathrm{z}, \mathrm{t})\right] / 2,
$$


where $E(z)$ is the Young modulus, $\sigma_{i j}$ is the stress tensor; $u_{i j}=\frac{1}{2}\left(\frac{\partial u_{i}}{\partial x_{j}}+\frac{\partial u_{j}}{\partial x_{i}}\right)$ is the deformation tensor; $u_{i}, u_{j}$ are the components $u_{x}(x, y, z, t), u_{y}(x, y, z, t)$ and $u_{z}(x, y, z, t)$ of the displacement vector $\vec{u}(x, y, z, t) ; x_{i}, x_{j}$ are the coordinate $x$, $y$, z. The Eq. (3) could be transform to the following form

$$
\begin{aligned}
& \mu(x, y, z, t)=\left[\frac{\partial u_{i}(x, y, z, t)}{\partial x_{j}}+\frac{\partial u_{j}(x, y, z, t)}{\partial x_{i}}\right]\left\{\frac{1}{2}\left[\frac{\partial u_{i}(x, y, z, t)}{\partial x_{j}}+\frac{\partial u_{j}(x, y, z, t)}{\partial x_{i}}\right]-\right. \\
& \left.-\varepsilon_{0} \delta_{i j}+\frac{\sigma(z) \delta_{i j}}{1-2 \sigma(z)}\left[\frac{\partial u_{k}(x, y, z, t)}{\partial x_{k}}-3 \varepsilon_{0}\right]-K(z) \beta(z)\left[T(x, y, z, t)-T_{0}\right] \delta_{i j}\right\} \frac{\Omega}{2} E(z)
\end{aligned}
$$

where $\sigma$ is Poisson coefficient; $\varepsilon_{0}=\left(a_{s}-a_{E L}\right) / a_{E L}$ is the mismatch parameter; $a_{s}, a_{E L}$ are the lattice distances of the substrate and the epitaxial layer; $K$ is the modulus of uniform compression; $\beta$ is the coefficient of thermal expansion; $T_{r}$ is the equilibrium temperature, which coincide (for our case) with room temperature. Components of displacement vector could be obtained by solution of the following systems of equations [25]

$$
\left\{\begin{array}{l}
\rho(z) \frac{\partial^{2} u_{x}(x, y, z, t)}{\partial t^{2}}=\frac{\partial \sigma_{x x}(x, y, z, t)}{\partial x}+\frac{\partial \sigma_{x y}(x, y, z, t)}{\partial y}+\frac{\partial \sigma_{x z}(x, y, z, t)}{\partial z} \\
\rho(z) \frac{\partial^{2} u_{y}(x, y, z, t)}{\partial t^{2}}=\frac{\partial \sigma_{y x}(x, y, z, t)}{\partial x}+\frac{\partial \sigma_{y y}(x, y, z, t)}{\partial y}+\frac{\partial \sigma_{y z}(x, y, z, t)}{\partial z} \\
\rho(z) \frac{\partial^{2} u_{z}(x, y, z, t)}{\partial t^{2}}=\frac{\partial \sigma_{z x}(x, y, z, t)}{\partial x}+\frac{\partial \sigma_{z y}(x, y, z, t)}{\partial y}+\frac{\partial \sigma_{z z}(x, y, z, t)}{\partial z} \\
\text { where } \quad \sigma_{i j}=\frac{E(z)}{2[1+\sigma(z)]}\left[\frac{\partial u_{i}(x, y, z, t)}{\partial x_{j}}+\frac{\partial u_{j}(x, y, z, t)}{\partial x_{i}}-\frac{\delta_{i j}}{3} \frac{\partial u_{k}(x, y, z, t)}{\partial x_{k}}\right]+K(z) \delta_{i j} \times
\end{array}\right.
$$
$\times \frac{\partial u_{k}(x, y, z, t)}{\partial x_{k}}-\beta(z) K(z)\left[T(x, y, z, t)-T_{r}\right], \rho(z)$ is the density of materials of heterostructure, $\delta_{i j}$ is the Kronecker symbol. With account the relation for $\sigma_{i j}$ last system of equation could be written as

$$
\begin{aligned}
& \rho(z) \frac{\partial^{2} u_{x}(x, y, z, t)}{\partial t^{2}}=\left\{K(z)+\frac{5 E(z)}{6[1+\sigma(z)]}\right\} \frac{\partial^{2} u_{x}(x, y, z, t)}{\partial x^{2}}+\left\{K(z)-\frac{E(z)}{3[1+\sigma(z)]}\right\} \times \\
& \times \frac{\partial^{2} u_{y}(x, y, z, t)}{\partial x \partial y}+\frac{E(z)}{2[1+\sigma(z)]}\left[\frac{\partial^{2} u_{y}(x, y, z, t)}{\partial y^{2}}+\frac{\partial^{2} u_{z}(x, y, z, t)}{\partial z^{2}}\right]+\left[K(z)+\frac{E(z)}{3[1+\sigma(z)]}\right] \times \\
& \times \frac{\partial^{2} u_{z}(x, y, z, t)}{\partial x \partial z}-K(z) \beta(z) \frac{\partial T(x, y, z, t)}{\partial x} \\
& \rho(z) \frac{\partial^{2} u_{y}(x, y, z, t)}{\partial t^{2}}=\frac{E(z)}{2[1+\sigma(z)]}\left[\frac{\partial^{2} u_{y}(x, y, z, t)}{\partial x^{2}}+\frac{\partial^{2} u_{x}(x, y, z, t)}{\partial x \partial y}\right]-\frac{\partial T(x, y, z, t)}{\partial y} \times
\end{aligned}
$$




$$
\begin{aligned}
& \times K(z) \beta(z)+\frac{\partial}{\partial z}\left\{\frac{E(z)}{2[1+\sigma(z)]}\left[\frac{\partial u_{y}(x, y, z, t)}{\partial z}+\frac{\partial u_{z}(x, y, z, t)}{\partial y}\right]\right\}+\frac{\partial^{2} u_{y}(x, y, z, t)}{\partial y^{2}} \times \\
& \times\left\{\frac{5 E(z)}{12[1+\sigma(z)]}+K(z)\right\}+\left\{K(z)-\frac{E(z)}{6[1+\sigma(z)]}\right\} \frac{\partial^{2} u_{y}(x, y, z, t)}{\partial y \partial z}+K(z) \frac{\partial^{2} u_{y}(x, y, z, t)}{\partial x \partial y} \\
& \rho(z) \frac{\partial^{2} u_{z}(x, y, z, t)}{\partial t^{2}}=\frac{E(z)}{2[1+\sigma(z)]}\left[\frac{\partial^{2} u_{z}(x, y, z, t)}{\partial x^{2}}+\frac{\partial^{2} u_{z}(x, y, z, t)}{\partial y^{2}}+\frac{\partial^{2} u_{x}(x, y, z, t)}{\partial x \partial z}+\right. \\
& \left.+\frac{\partial^{2} u_{y}(x, y, z, t)}{\partial y \partial z}\right]+\frac{\partial}{\partial z}\left\{K(z)\left[\frac{\partial u_{x}(x, y, z, t)}{\partial x}+\frac{\partial u_{y}(x, y, z, t)}{\partial y}+\frac{\partial u_{x}(x, y, z, t)}{\partial z}\right]\right\}+ \\
& +\frac{1}{6} \frac{\partial}{\partial z}\left\{\frac{E(z)}{1+\sigma(z)}\left[6 \frac{\partial u_{z}(x, y, z, t)}{\partial z}-\frac{\partial u_{x}(x, y, z, t)}{\partial x}-\frac{\partial u_{y}(x, y, z, t)}{\partial y}-\frac{\partial u_{z}(x, y, z, t)}{\partial z}\right]\right\}- \\
& -K(z) \beta(z) \frac{\partial T(x, y, z, t)}{\partial z}
\end{aligned}
$$

Conditions for the system of Eq. (8) could be written in the form

$$
\begin{gathered}
\frac{\partial \vec{u}(0, y, z, t)}{\partial x}=0 \quad \frac{\partial \vec{u}\left(L_{x}, y, z, t\right)}{\partial x}=0 \quad \frac{\partial \vec{u}(x, 0, z, t)}{\partial y}=0 \quad \frac{\partial \vec{u}\left(x, L_{y}, z, t\right)}{\partial y}=0 \\
\frac{\partial \vec{u}(x, y, 0, t)}{\partial z}=0 \quad \frac{\partial \vec{u}\left(x, y, L_{z}, t\right)}{\partial z}=0 ; \vec{u}(x, y, z, 0)=\vec{u}_{0} ; \vec{u}(x, y, z, \infty)=\vec{u}_{0} .
\end{gathered}
$$

We determine spatio-temporal distributions of concentrations of dopant and radiation defects by solving the Eqs.(1), (3) and (5) framework standard method of averaging of function corrections [26]. Previously we transform the Eqs.(1), (3) and (5) to the following form with account initial distributions of the considered concentrations

$$
\begin{gathered}
\frac{\partial C(x, y, z, t)}{\partial t}=\frac{\partial}{\partial x}\left[D \frac{\partial C(x, y, z, t)}{\partial x}\right]+\frac{\partial}{\partial y}\left[D \frac{\partial C(x, y, z, t)}{\partial y}\right]+\frac{\partial}{\partial z}\left[D \frac{\partial C(x, y, z, t)}{\partial z}\right]+ \\
+f_{C}(x, y, z) \delta(t)+\Omega \frac{\partial}{\partial x}\left[\frac{D_{S}}{k T} \nabla_{S} \mu(x, y, z, t) \int_{0}^{L_{z}} C(x, y, W, t) d W\right]+ \\
+\Omega \frac{\partial}{\partial y}\left[\frac{D_{S}}{k T} \nabla_{S} \mu(x, y, z, t) \int_{0}^{L_{z}} C(x, y, W, t) d W\right] \\
\frac{\partial I(x, y, z, t)}{\partial t}=\frac{\partial}{\partial x}\left[D_{I}(x, y, z, T) \frac{\partial I(x, y, z, t)}{\partial x}\right]+\frac{\partial}{\partial y}\left[D_{I}(x, y, z, T) \frac{\partial I(x, y, z, t)}{\partial y}\right]+ \\
+\frac{\partial}{\partial z}\left[D_{I}(x, y, z, T) \frac{\partial I(x, y, z, t)}{\partial z}\right]+\Omega \frac{\partial}{\partial x}\left[\frac{D_{I S}}{k T} \nabla_{S} \mu_{1}(x, y, z, t) \int_{0}^{L_{z}} I(x, y, W, t) d W\right]+
\end{gathered}
$$




$$
\begin{aligned}
& +\Omega \frac{\partial}{\partial y}\left[\frac{D_{I S}}{k T} \nabla_{S} \mu_{1}(x, y, z, t) \int_{0}^{L_{z}} I(x, y, W, t) d W\right]-k_{I, I}(x, y, z, T) I^{2}(x, y, z, t)- \\
& -k_{I, V}(x, y, z, T) I(x, y, z, t) V(x, y, z, t)+f_{I}(x, y, z) \delta(t) \\
& \frac{\partial V(x, y, z, t)}{\partial t}=\frac{\partial}{\partial x}\left[D_{V}(x, y, z, T) \frac{\partial V(x, y, z, t)}{\partial x}\right]+\frac{\partial}{\partial y}\left[D_{V}(x, y, z, T) \frac{\partial V(x, y, z, t)}{\partial y}\right]+ \\
& +\frac{\partial}{\partial z}\left[D_{V}(x, y, z, T) \frac{\partial V(x, y, z, t)}{\partial z}\right]+\Omega \frac{\partial}{\partial x}\left[\frac{D_{V S}}{k T} \nabla_{S} \mu_{1}(x, y, z, t) \int_{0}^{L_{z}} V(x, y, W, t) d W\right]+ \\
& +\Omega \frac{\partial}{\partial y}\left[\frac{D_{V S}}{k T} \nabla_{S} \mu_{1}(x, y, z, t) \int_{0}^{L_{z}} V(x, y, W, t) d W\right]-k_{V, V}(x, y, z, T) V^{2}(x, y, z, t)- \\
& -k_{I, V}(x, y, z, T) I(x, y, z, t) V(x, y, z, t)+f_{V}(x, y, z) \delta(t) \\
& \frac{\partial \Phi_{I}(x, y, z, t)}{\partial t}=\frac{\partial}{\partial x}\left[D_{\Phi_{I}}(x, y, z, T) \frac{\partial \Phi_{I}(x, y, z, t)}{\partial x}\right]+\frac{\partial}{\partial y}\left[D_{\Phi_{I}}(x, y, z, T) \frac{\partial \Phi_{I}(x, y, z, t)}{\partial y}\right]+ \\
& +\frac{\partial}{\partial z}\left[D_{\Phi_{I}}(x, y, z, T) \frac{\partial \Phi_{I}(x, y, z, t)}{\partial z}\right]+\Omega \frac{\partial}{\partial x}\left[\frac{D_{\Phi_{I} S}}{k T} \nabla_{S} \mu_{1}(x, y, z, t) \int_{0}^{L_{z}} \Phi_{I}(x, y, W, t) d W\right]+ \\
& +\Omega \frac{\partial}{\partial y}\left[\frac{D_{\Phi_{I} S}}{k T} \nabla_{S} \mu_{1}(x, y, z, t) \int_{0}^{L_{z}} \Phi_{I}(x, y, W, t) d W\right]+k_{I}(x, y, z, T) I(x, y, z, t)+ \\
& +k_{I, I}(x, y, z, T) I^{2}(x, y, z, t)+f_{\Phi_{I}}(x, y, z) \delta(t) \\
& \frac{\partial \Phi_{V}(x, y, z, t)}{\partial t}=\frac{\partial}{\partial x}\left[D_{\Phi_{V}}(x, y, z, T) \frac{\partial \Phi_{V}(x, y, z, t)}{\partial x}\right]+\frac{\partial}{\partial y}\left[D_{\Phi_{V}}(x, y, z, T) \frac{\partial \Phi_{V}(x, y, z, t)}{\partial y}\right]+ \\
& +\frac{\partial}{\partial z}\left[D_{\Phi_{V}}(x, y, z, T) \frac{\partial \Phi_{V}(x, y, z, t)}{\partial z}\right]+\Omega \frac{\partial}{\partial x}\left[\frac{D_{\Phi_{V} S}}{k T} \nabla_{S} \mu_{1}(x, y, z, t) \int_{0}^{L_{z}} \Phi_{V}(x, y, W, t) d W\right]+ \\
& +\Omega \frac{\partial}{\partial y}\left[\frac{D_{\Phi_{V} S}}{k T} \nabla_{S} \mu_{1}(x, y, z, t) \int_{0}^{L_{z}} \Phi_{V}(x, y, W, t) d W\right]+k_{V}(x, y, z, T) V(x, y, z, t)+ \\
& +k_{V, V}(x, y, z, T) V^{2}(x, y, z, t)+f_{\Phi_{V}}(x, y, z) \delta(t)
\end{aligned}
$$

Now we replace concentrations of dopant and radiation defects in the right sides of Eqs. (1a), (3a) and (5a) on their not yet known average values $\alpha_{1 \rho}$. In this situation we obtain equations for the first-order approximations of the required concentrations in the following form

$$
\frac{\partial C_{1}(x, y, z, t)}{\partial t}=\alpha_{1 C} \Omega \frac{\partial}{\partial x}\left[z \frac{D_{S}}{k T} \nabla_{s} \mu_{1}(x, y, z, t)\right]+\alpha_{1 C} \Omega \frac{\partial}{\partial y}\left[z \frac{D_{S}}{k T} \nabla_{s} \mu_{1}(x, y, z, t)\right]+
$$




$$
\begin{gathered}
+f_{C}(x, y, z) \delta(t) \\
\frac{\partial I_{1}(x, y, z, t)}{\partial t}=\alpha_{1 I} z \Omega \frac{\partial}{\partial x}\left[\frac{D_{I S}}{k T} \nabla_{S} \mu(x, y, z, t)\right]+\alpha_{1 I} \Omega \frac{\partial}{\partial y}\left[z \frac{D_{I S}}{k T} \nabla_{S} \mu(x, y, z, t)\right]+ \\
+f_{I}(x, y, z) \delta(t)-\alpha_{1 I}^{2} k_{I, I}(x, y, z, T)-\alpha_{1 I} \alpha_{1 V} k_{I, V}(x, y, z, T) \\
+f_{V}(x, y, z) \delta(t)-\alpha_{1 V}^{2} k_{V, V}(x, y, z, T)-\alpha_{1 I} \alpha_{1 V} k_{I, V}(x, y, z, T) \\
\frac{\partial V_{1}(x, y, z, t)}{\partial t}=\alpha_{1 V} z \Omega \frac{\partial}{\partial x}\left[\frac{D_{V S}}{k T} \nabla_{S} \mu_{1}(x, y, z, t)\right]+\alpha_{1 V} \Omega \frac{\partial}{\partial y}\left[z \frac{D_{V S}}{k T} \nabla_{S} \mu_{1}(x, y, z, t)\right]+ \\
\frac{\partial t}{\partial t}=\alpha_{1 \Phi_{I}} z \Omega \frac{\partial}{\partial x}\left[\frac{D_{\Phi_{I} S}}{k T} \nabla_{S} \mu_{1}(x, y, z, t)\right]+\alpha_{1 \Phi_{I}} z \Omega \frac{\partial}{\partial y}\left[\frac{D_{\Phi_{I} S}}{k T} \nabla_{S} \mu_{1}(x, y, z, t)\right]+ \\
\frac{\partial \Phi_{1 V}(x, y, z, t)}{\partial t}=\alpha_{1 \Phi_{V}} z \Omega \frac{\partial}{\partial x}\left[\frac{D_{\Phi_{V} S}}{k T} \nabla_{S} \mu_{1}(x, y, z, t)\right]+\alpha_{1 \Phi_{V}} z \Omega \frac{\partial}{\partial y}\left[\frac{D_{\Phi_{V} S}}{k T} \nabla_{S} \mu_{1}(x, y, z, t)\right]+ \\
+f_{\Phi_{V}}(x, y, z) \delta(t)+k_{V}(x, y, z, T) V(x, y, z, t)+k_{V, V}(x, y, z, T) V^{2}(x, y, z, t)
\end{gathered}
$$

Integration of the left and right sides of the Eqs. $(1 b),(3 b)$ and $(5 b)$ on time, gives us possibility to obtain relations for above approximation in the final form

$$
\begin{gathered}
C_{1}(x, y, z, t)=\alpha_{1 C} \Omega \frac{\partial}{\partial x} \int_{0}^{t} D_{S L}(x, y, z, T) \frac{z}{k T}\left[1+\varsigma_{1} \frac{V(x, y, z, \tau)}{V^{*}}+\varsigma_{2} \frac{V^{2}(x, y, z, \tau)}{\left(V^{*}\right)^{2}}\right] \times \\
\left.\times \nabla_{S} \mu_{1}(x, y, z, \tau)\left[1+\frac{\xi_{S} \alpha_{1 C}^{\gamma}}{P^{\gamma}(x, y, z, T)}\right] d \tau\right\}+\alpha_{1 C} \frac{\partial}{\partial y} \int_{0}^{t} D_{S L}(x, y, z, T)\left[1+\frac{\xi_{s} \alpha_{1 C}^{\gamma}}{P^{\gamma}(x, y, z, T)}\right]+ \\
\times \Omega \nabla_{s} \mu_{1}(x, y, z, \tau) \frac{z}{k T}\left[1+\varsigma_{1} \frac{V(x, y, z, \tau)}{V^{*}}+\varsigma_{2} \frac{V^{2}(x, y, z, \tau)}{\left(V^{*}\right)^{2}}\right] d \tau+f_{C}(x, y, z) \\
I_{1}(x, y, z, t)=\alpha_{1 I} z \Omega \frac{\partial}{\partial x} \int_{0}^{t} \frac{D_{I S}}{k T} \nabla_{S} \mu_{1}(x, y, z, \tau) d \tau+\alpha_{1 I} z \Omega \frac{\partial}{\partial y} \int_{0}^{t} \frac{D_{I S}}{k T} \nabla_{S} \mu_{1}(x, y, z, \tau) d \tau+ \\
+f_{I}(x, y, z)-\alpha_{1 I}^{2} \int_{0}^{t} k_{I, I}(x, y, z, T) d \tau-\alpha_{1 I} \alpha_{1 V} \int_{0}^{t} k_{I, V}(x, y, z, T) d \tau \\
V_{1}(x, y, z, t)=\alpha_{1 V} z \Omega \frac{\partial}{\partial x} \int_{0}^{t} \frac{D_{I S}}{k T} \nabla_{S} \mu_{1}(x, y, z, \tau) d \tau+\alpha_{1 V} z \Omega \frac{\partial}{\partial y} \int_{0}^{t} \frac{D_{I S}}{k T} \nabla_{S} \mu_{1}(x, y, z, \tau) d \tau+ \\
+f_{V}(x, y, z)-\alpha_{1 V}^{2} \int_{0}^{t} k_{V, V}(x, y, z, T) d \tau-\alpha_{1 I} \alpha_{1 V} \int_{0}^{t} k_{I, V}(x, y, z, T) d \tau
\end{gathered}
$$




$$
\begin{aligned}
& \Phi_{1 I}(x, y, z, t)=\alpha_{1 \Phi_{I}} z \Omega \frac{\partial}{\partial x} \int_{0}^{t} \frac{D_{\Phi_{I} S}}{k T} \nabla_{S} \mu_{1}(x, y, z, \tau) d \tau+\Omega \frac{\partial}{\partial x} \int_{0}^{t} \frac{D_{\Phi_{I} S}}{k T} \nabla_{S} \mu_{1}(x, y, z, \tau) d \tau \times \\
& \quad \times \alpha_{1 \Phi_{I}} z+f_{\Phi_{I}}(x, y, z)++\int_{0}^{t} k_{I}(x, y, z, T) I(x, y, z, \tau) d \tau+\int_{0}^{t} k_{I, I}(x, y, z, T) I^{2}(x, y, z, \tau) d \tau \quad \text { (5c) } \\
& \Phi_{1 V}(x, y, z, t)=\alpha_{1 \Phi_{V}} z \Omega \frac{\partial}{\partial x} \int_{0}^{t} \frac{D_{\Phi_{V} S}}{k T} \nabla_{S} \mu_{1}(x, y, z, \tau) d \tau+\Omega \frac{\partial}{\partial x} \int_{0}^{t} \frac{D_{\Phi_{V} S}}{k T} \nabla_{S} \mu_{1}(x, y, z, \tau) d \tau \times \\
& \quad \times \alpha_{1 \Phi_{V}} z+f_{\Phi_{V}}(x, y, z)+\int_{0}^{t} k_{V}(x, y, z, T) V(x, y, z, \tau) d \tau+\int_{0}^{t} k_{V, V}(x, y, z, T) V^{2}(x, y, z, \tau) d \tau
\end{aligned}
$$

We calculate the average values of the first-order approximations of concentrations of dopant and radiation defects by using the following standard relation [26]

$$
\alpha_{1 \rho}=\frac{1}{\Theta L_{x} L_{y} L_{z}} \int_{0}^{\Theta} \int_{0}^{L_{x}} \int_{0}^{L_{y} L_{z}} \int_{0} \rho_{1}(x, y, z, t) d z d y d x d t .
$$

Substitution of the relations (1c), (3c) and (5c) into relation (9) gives us possibility to obtain required average values in the following form

$$
\begin{aligned}
\alpha_{1 C} & =\frac{1}{L_{x} L_{y} L_{z}} \int_{0}^{L_{x}} \int_{0}^{L_{y}} \int_{0}^{L_{z}} f_{C}(x, y, z) d z d y d x \quad \alpha_{1 I}=\sqrt{\frac{\left(a_{3}+A\right)^{2}}{4 a_{4}^{2}}-4\left(B+\frac{\Theta a_{3} B+\Theta^{2} L_{x} L_{y} L_{z} a_{1}}{a_{4}}\right)}- \\
& -\frac{a_{3}+A}{4 a_{4}}, \alpha_{1 V}=\frac{1}{S_{I V 00}}\left[\frac{\Theta}{\alpha_{1 I}} \int_{0}^{L_{x}} \int_{0}^{L_{y} L_{z}} \int_{0} f_{I}(x, y, z) d z d y d x-\alpha_{1 I} S_{I I 00}-\Theta L_{x} L_{y} L_{z}\right]
\end{aligned}
$$

where $S_{\rho \rho i j}=\int_{0}^{\Theta}(\Theta-t) \int_{0}^{L_{x}} \int_{0}^{L_{y} L_{z}} \int_{0} k_{\rho, \rho}(x, y, z, T) I_{1}^{i}(x, y, z, t) V_{1}^{j}(x, y, z, t) d z d y d x d t, a_{4}=S_{I I 00} \times$

$$
\times\left(S_{I V 00}^{2}-S_{I I 00} S_{V V 00}\right) \quad, \quad a_{3}=S_{I V 00} S_{I I 00}+S_{I V 00}^{2}-S_{I I 00} S_{V V 00}
$$$$
a_{2}=\int_{0}^{L_{x}} \int_{0}^{L_{y} L_{z}} \int_{0} f_{V}(x, y, z) d z d y d x \times
$$

$\times S_{I V 00} S_{I V 00}^{2}+S_{I V 00} \Theta L_{x}^{2} L_{y}^{2} L_{z}^{2}+2 S_{V V 00} S_{I I 00} \int_{0}^{L_{x}} \int_{0}^{L_{y} L_{z}} f_{0} f_{I}(x, y, z) d z d y d x-\Theta L_{x}^{2} L_{y}^{2} L_{z}^{2} S_{V V 00}-$

$-S_{I V 00}^{2} \int_{0}^{L_{x}} \int_{0}^{L_{y} L_{z}} \int_{0} f_{I}(x, y, z) d z d y d x, a_{1}=S_{I V 00} \int_{0}^{L_{x}} \int_{0}^{L_{y} L_{z}} \int_{0}^{L_{I}} f_{I}(x, y, z) d z d y d x, a_{0}=S_{V V 00} \times$

$$
\times\left[\int_{0}^{L_{x}} \int_{0}^{L_{y} \int_{z}} f_{0}(x, y, z) d z d y d x\right]^{2} \quad, \quad A=\sqrt{8 y+\Theta^{2} \frac{a_{3}^{2}}{a_{4}^{2}}-4 \Theta \frac{a_{2}}{a_{4}}}
$$

$B=\frac{\Theta a_{2}}{6 a_{4}}+\sqrt[3]{\sqrt{q^{2}+p^{3}}-q}-$

$$
-\sqrt[3]{\sqrt{q^{2}+p^{3}}+q}, q=\frac{\Theta^{3} a_{2}}{24 a_{4}^{2}}\left(4 a_{0}-\Theta L_{x} L_{y} L_{z} \frac{a_{1} a_{3}}{a_{4}}\right)-\Theta^{2} \frac{a_{0}}{8 a_{4}^{2}}\left(4 \Theta a_{2}-\Theta^{2} \frac{a_{3}^{2}}{a_{4}}\right)-
$$




$$
\begin{aligned}
& -\frac{\Theta^{3} a_{2}^{3}}{54 a_{4}^{3}}-L_{x}^{2} L_{y}^{2} L_{z}^{2} \frac{\Theta^{4} a_{1}^{2}}{8 a_{4}^{2}}, p=\Theta^{2} \frac{4 a_{0} a_{4}-\Theta L_{x} L_{y} L_{z} a_{1} a_{3}}{12 a_{4}^{2}}-\frac{\Theta a_{2}}{18 a_{4}}, \\
& \alpha_{1 \Phi_{I}}=\frac{R_{I 1}}{\Theta L_{x} L_{y} L_{z}}+\frac{S_{I I 20}}{\Theta L_{x} L_{y} L_{z}}+\frac{1}{L_{x} L_{y} L_{z}} \int_{0}^{L_{x} L_{y} L_{z}} \int_{0} \int_{0} f_{\Phi_{I}}(x, y, z) d z d y d x \\
& \alpha_{1 \Phi_{V}}=\frac{R_{V 1}}{\Theta L_{x} L_{y} L_{z}}+\frac{S_{V V 20}}{\Theta L_{x} L_{y} L_{z}}+\frac{1}{L_{x} L_{y} L_{z}} \int_{0}^{L_{x} L_{y} L_{z}} \int_{0} \int_{0} f_{\Phi_{V}}(x, y, z) d z d y d x,
\end{aligned}
$$

where $R_{\rho i}=\int_{0}^{\Theta}(\Theta-t) \int_{0}^{L_{x}} \int_{0}^{L_{y} L_{z}} \int_{0} k_{I}(x, y, z, T) I_{1}^{i}(x, y, z, t) d z d y d x d t$.

We determine approximations of the second and higher orders of concentrations of dopant and radiation defects framework standard iterative procedure of method of averaging of function corrections [26]. Framework this procedure to determine approximations of the $n$-th order of concentrations of dopant and radiation defects we replace the required concentrations in the Eqs. (1c), (3c), (5c) on the following sum $\alpha_{n} \rho^{+} \rho_{n-1}(x, y, z, t)$. The replacement leads to the following transformation of the appropriate equations

$$
\begin{aligned}
& \frac{\partial C_{2}(x, y, z, t)}{\partial t}=\frac{\partial}{\partial x}\left(\left\{1+\xi \frac{\left[\alpha_{2 C}+C_{1}(x, y, z, t)\right]^{\gamma}}{P^{\gamma}(x, y, z, T)}\right\}\left[1+\varsigma_{1} \frac{V(x, y, z, t)}{V^{*}}+\varsigma_{2} \frac{V^{2}(x, y, z, t)}{\left(V^{*}\right)^{2}}\right] \times\right. \\
& \left.\times D_{L}(x, y, z, T) \frac{\partial C_{1}(x, y, z, t)}{\partial x}\right)+\frac{\partial}{\partial y}\left(\left[1+\varsigma_{1} \frac{V(x, y, z, t)}{V^{*}}+\varsigma_{2} \frac{V^{2}(x, y, z, t)}{\left(V^{*}\right)^{2}}\right] \frac{\partial C_{1}(x, y, z, t)}{\partial y} \times\right. \\
& \left.\times D_{L}(x, y, z, T)\left\{1+\xi \frac{\left[\alpha_{2 C}+C_{1}(x, y, z, t)\right]^{\gamma}}{P^{\gamma}(x, y, z, T)}\right\}\right) \frac{\partial}{\partial z}\left(\left[1+\varsigma_{1} \frac{V(x, y, z, t)}{V^{*}}+\varsigma_{2} \frac{V^{2}(x, y, z, t)}{\left(V^{*}\right)^{2}}\right] \times\right. \\
& \left.\times D_{L}(x, y, z, T) \frac{\partial C_{1}(x, y, z, t)}{\partial z}\left\{1+\xi \frac{\left[\alpha_{2 C}+C_{1}(x, y, z, t)\right]^{\gamma}}{P^{\gamma}(x, y, z, T)}\right\}\right)+f_{C}(x, y, z) \delta(t)+ \\
& +\Omega \frac{\partial}{\partial x}\left\{\frac{D_{S}}{k T} \nabla_{S} \mu_{1}(x, y, z, t) \int_{0}^{L_{z}}\left[\alpha_{2 C}+C(x, y, W, t)\right] d W\right\}+ \\
& +\Omega \frac{\partial}{\partial y}\left\{\frac{D_{S}}{k T} \nabla_{S} \mu_{1}(x, y, z, t) \int_{0}^{L_{2}}\left[\alpha_{2 C}+C(x, y, W, t)\right] d W\right\} \\
& \frac{\partial I_{2}(x, y, z, t)}{\partial t}=\frac{\partial}{\partial x}\left[D_{I}(x, y, z, T) \frac{\partial I_{1}(x, y, z, t)}{\partial x}\right]+\frac{\partial}{\partial y}\left[D_{I}(x, y, z, T) \frac{\partial I_{1}(x, y, z, t)}{\partial y}\right]+ \\
& +\frac{\partial}{\partial z}\left[D_{I}(x, y, z, T) \frac{\partial I_{1}(x, y, z, t)}{\partial z}\right]-k_{I, I}(x, y, z, T)\left[\alpha_{1 I}+I_{1}(x, y, z, t)\right]^{2}-k_{I, V}(x, y, z, T) \times \\
& \times\left[\alpha_{1 I}+I_{1}(x, y, z, t)\right]\left[\alpha_{1 V}+V_{1}(x, y, z, t)\right]+\Omega \frac{\partial}{\partial x}\left\{\nabla_{S} \mu(x, y, z, t) \int_{0}^{L_{z}}\left[\alpha_{2 I}+I_{1}(x, y, W, t)\right] d W \times\right.
\end{aligned}
$$




$$
\begin{aligned}
& \left.\times \frac{D_{I S}}{k T}\right\}+\Omega \frac{\partial}{\partial y}\left\{\frac{D_{I S}}{k T} \nabla_{s} \mu(x, y, z, t) \int_{0}^{L_{z}}\left[\alpha_{2 I}+I_{1}(x, y, W, t)\right] d W\right\} \\
& \frac{\partial V_{2}(x, y, z, t)}{\partial t}=\frac{\partial}{\partial x}\left[D_{V}(x, y, z, T) \frac{\partial V_{1}(x, y, z, t)}{\partial x}\right]+\frac{\partial}{\partial y}\left[D_{V}(x, y, z, T) \frac{\partial V_{1}(x, y, z, t)}{\partial y}\right]+ \\
& +\frac{\partial}{\partial z}\left[D_{V}(x, y, z, T) \frac{\partial V_{1}(x, y, z, t)}{\partial z}\right]-k_{V, V}(x, y, z, T)\left[\alpha_{1 V}+V_{1}(x, y, z, t)\right]^{2}-k_{I, V}(x, y, z, T) \times \\
& \times\left[\alpha_{1 I}+I_{1}(x, y, z, t)\right]\left[\alpha_{1 V}+V_{1}(x, y, z, t)\right]+\Omega \frac{\partial}{\partial x}\left\{\nabla_{s} \mu(x, y, z, t) \int_{0}^{L_{z}}\left[\alpha_{2 V}+V_{1}(x, y, W, t)\right] d W \times\right. \\
& \left.\times \frac{D_{V S}}{k T}\right\}+\Omega \frac{\partial}{\partial y}\left\{\frac{D_{V S}}{k T} \nabla_{s} \mu(x, y, z, t) \int_{0}^{L_{z}}\left[\alpha_{2 V}+V_{1}(x, y, W, t)\right] d W\right\} \\
& \frac{\partial \Phi_{2 I}(x, y, z, t)}{\partial t}=\frac{\partial}{\partial x}\left[D_{\Phi_{I}}(x, y, z, T) \frac{\partial \Phi_{1 I}(x, y, z, t)}{\partial x}\right]+\frac{\partial}{\partial y}\left[D_{\Phi_{I}}(x, y, z, T) \frac{\partial \Phi_{1 I}(x, y, z, t)}{\partial y}\right]+ \\
& +\Omega \frac{\partial}{\partial x}\left\{\frac{D_{\Phi_{I} S}}{k T} \nabla_{S} \mu(x, y, z, t) \int_{0}^{L_{z}}\left[\alpha_{2 \Phi_{I}}+\Phi_{1 I}(x, y, W, t)\right] d W\right\}+k_{I, I}(x, y, z, T) I^{2}(x, y, z, t)+ \\
& +\Omega \frac{\partial}{\partial y}\left\{\frac{D_{\Phi_{I} S}}{k T} \nabla_{S} \mu(x, y, z, t) \int_{0}^{L_{z}}\left[\alpha_{2 \Phi_{I}}+\Phi_{1 I}(x, y, W, t)\right] d W\right\}+k_{I}(x, y, z, T) I(x, y, z, t)+ \\
& +\frac{\partial}{\partial z}\left[D_{\Phi_{I}}(x, y, z, T) \frac{\partial \Phi_{1 I}(x, y, z, t)}{\partial z}\right]+f_{\Phi_{I}}(x, y, z) \delta(t) \\
& \frac{\partial \Phi_{2 V}(x, y, z, t)}{\partial t}=\frac{\partial}{\partial x}\left[D_{\Phi_{V}}(x, y, z, T) \frac{\partial \Phi_{1 V}(x, y, z, t)}{\partial x}\right]+\frac{\partial}{\partial y}\left[D_{\Phi_{V}}(x, y, z, T) \frac{\partial \Phi_{1 V}(x, y, z, t)}{\partial y}\right]+ \\
& +\Omega \frac{\partial}{\partial x}\left\{\frac{D_{\Phi_{V} S}}{k T} \nabla_{S} \mu(x, y, z, t) \int_{0}^{L_{z}}\left[\alpha_{2 \Phi_{V}}+\Phi_{1 V}(x, y, W, t)\right] d W\right\}+k_{V, V}(x, y, z, T) V^{2}(x, y, z, t)+ \\
& +\Omega \frac{\partial}{\partial y}\left\{\frac{D_{\Phi_{V} S}}{k T} \nabla_{S} \mu(x, y, z, t) \int_{0}^{L_{z}}\left[\alpha_{2 \Phi_{V}}+\Phi_{1 V}(x, y, W, t)\right] d W\right\}+k_{V}(x, y, z, T) V(x, y, z, t)+ \\
& +\frac{\partial}{\partial z}\left[D_{\Phi_{V}}(x, y, z, T) \frac{\partial \Phi_{1 V}(x, y, z, t)}{\partial z}\right]+f_{\Phi_{V}}(x, y, z) \delta(t)
\end{aligned}
$$

Integration of the left and the right sides of Eqs. (1d), $(3 d)$ and $(5 d)$ gives us possibility to obtain relations for the required concentrations in the final form

$$
C_{2}(x, y, z, t)=\frac{\partial}{\partial x} \int_{0}^{t}\left\{1+\xi \frac{\left[\alpha_{2 C}+C_{1}(x, y, z, \tau)\right]^{\gamma}}{P^{\gamma}(x, y, z, T)}\right\}\left[1+\varsigma_{1} \frac{V(x, y, z, \tau)}{V^{*}}+\varsigma_{2} \frac{V^{2}(x, y, z, \tau)}{\left(V^{*}\right)^{2}}\right] \times
$$




$$
\begin{aligned}
& \times D_{L}(x, y, z, T) \frac{\partial C_{1}(x, y, z, \tau)}{\partial x} d \tau+\frac{\partial}{\partial y} \int_{0}^{t} D_{L}(x, y, z, T)\left[1+\varsigma_{1} \frac{V(x, y, z, \tau)}{V^{*}}+\varsigma_{2} \frac{V^{2}(x, y, z, \tau)}{\left(V^{*}\right)^{2}}\right] \times \\
& \times \frac{\partial C_{1}(x, y, z, \tau)}{\partial y}\left\{1+\xi \frac{\left[\alpha_{2 C}+C_{1}(x, y, z, t)\right]^{\gamma}}{P^{\gamma}(x, y, z, T)}\right\}+\frac{\partial}{\partial z} \int_{0}^{t}\left[1+\varsigma_{1} \frac{V(x, y, z, \tau)}{V^{*}}+\varsigma_{2} \frac{V^{2}(x, y, z, \tau)}{\left(V^{*}\right)^{2}}\right] \times \\
& \times D_{L}(x, y, z, T) \frac{\partial C_{1}(x, y, z, \tau)}{\partial z}\left\{1+\xi \frac{\left[\alpha_{2 C}+C_{1}(x, y, z, \tau)\right]}{P^{\gamma}(x, y, z, T)}\right\} d \tau+f_{C}(x, y, z)+ \\
& +\Omega \frac{\partial}{\partial x} \int_{0}^{t} \frac{D_{S}}{k T} \nabla_{S} \mu(x, y, z, \tau) \int_{0}^{L_{z}}\left[\alpha_{2 C}+C_{1}(x, y, W, \tau)\right] d W d \tau+\frac{\partial}{\partial y} \int_{0}^{t} \nabla_{S} \mu(x, y, z, \tau) \times \\
& \times I_{2}(x, y, z, t)=\frac{\partial}{\partial x} \int_{0}^{t} D_{I}(x, y, z, T) \frac{\partial I_{1}(x, y, z, \tau)}{\partial x} d \tau \int_{0}^{L_{z}}\left[\alpha_{2 C}+C_{1}(x, y, W, \tau)\right] d W d \tau \\
& +\frac{\partial}{\partial z} \int_{0}^{t} D_{I}(x, y, z, T) \frac{\partial I_{1}(x, y, z, \tau)}{\partial z} d \tau-\int_{0}^{t} k_{I, I}(x, y, y, z, T) \frac{\partial I_{1}(x, y, z, \tau)}{\partial y} d \tau+ \\
& -\int_{0}^{t} k_{I, V}(x, y, z, T)\left[\alpha_{2 I}+I_{1}(x, y, z, \tau)\right]\left[\alpha_{2 V}+V_{1}(x, y, z, \tau)\right] d \tau+\frac{\partial}{\partial x} \int_{0}^{t} \nabla_{S} \mu(x, y, z, \tau) \times \\
& \times \Omega \frac{D_{I S}}{k T} \int_{0}^{L_{z}}\left[\alpha_{2 I}+I_{1}(x, y, W, \tau)\right] d W d \tau+\frac{\partial}{\partial y} \int_{0}^{t} \nabla_{S} \mu(x, y, z, \tau) \int_{0}^{L_{L}}\left[\alpha_{2 I}+I_{1}(x, y, W, \tau)\right] \times \\
& \times \Omega \frac{D_{I S}}{k T} d W d \tau
\end{aligned}
$$$$
V_{2}(x, y, z, t)=\frac{\partial}{\partial x} \int_{0}^{t} D_{V}(x, y, z, T) \frac{\partial V_{1}(x, y, z, \tau)}{\partial x} d \tau+\frac{\partial}{\partial y} \int_{0}^{t} D_{V}(x, y, z, T) \frac{\partial V_{1}(x, y, z, \tau)}{\partial y} d \tau+
$$$$
+\frac{\partial}{\partial z} \int_{0}^{t} D_{V}(x, y, z, T) \frac{\partial V_{1}(x, y, z, \tau)}{\partial z} d \tau-\int_{0}^{t} k_{V, V}(x, y, z, T)\left[\alpha_{2 V}+V_{1}(x, y, z, \tau)\right]^{2} d \tau-
$$$$
-\int_{0}^{t} k_{I, V}(x, y, z, T)\left[\alpha_{2 I}+I_{1}(x, y, z, \tau)\right]\left[\alpha_{2 V}+V_{1}(x, y, z, \tau)\right] d \tau+\frac{\partial}{\partial x} \int_{0}^{t} \nabla_{S} \mu(x, y, z, \tau) \times
$$$$
\times \Omega \frac{D_{V S}}{k T} \int_{0}^{L_{z}}\left[\alpha_{2 V}+V_{1}(x, y, W, \tau)\right] d W d \tau+\frac{\partial}{\partial y} \int_{0}^{t} \nabla_{S} \mu(x, y, z, \tau) \int_{0}^{L_{z}}\left[\alpha_{2 V}+V_{1}(x, y, W, \tau)\right] \times
$$$$
\times \Omega \frac{D_{V S}}{k T} d W d \tau+f_{V}(x, y, z)
$$ 


$$
\begin{aligned}
& \Phi_{2 I}(x, y, z, t)=\frac{\partial}{\partial x} \int_{0}^{t} D_{\Phi_{I}}(x, y, z, T) \frac{\partial \Phi_{1 I}(x, y, z, \tau)}{\partial x} d \tau+\frac{\partial}{\partial y} \int_{0}^{t} \frac{\partial \Phi_{1 I}(x, y, z, \tau)}{\partial y} \times \\
& \times D_{\Phi_{I}}(x, y, z, T) d \tau+\frac{\partial}{\partial z} \int_{0}^{t} D_{\Phi_{I}}(x, y, z, T) \frac{\partial \Phi_{1 I}(x, y, z, \tau)}{\partial z} d \tau+\Omega \frac{\partial}{\partial x} \int_{0}^{t} \nabla_{S} \mu(x, y, z, \tau) \times \\
& \times \frac{D_{\Phi_{I} S} S}{k T} \int_{0}^{L_{z}}\left[\alpha_{2 \Phi_{I}}+\Phi_{1 I}(x, y, W, \tau)\right] d W d \tau+\Omega \frac{\partial}{\partial y} \int_{0}^{t} \frac{D_{\Phi_{I} S}}{k T} \int_{0}^{L_{z}}\left[\alpha_{2 \Phi_{I}}+\Phi_{1 I}(x, y, W, \tau)\right] d W \times \\
& \times \nabla_{s} \mu(x, y, z, \tau) d \tau+\int_{0}^{t} k_{I, I}(x, y, z, T) I^{2}(x, y, z, \tau) d \tau+\int_{0}^{t} k_{I}(x, y, z, T) I(x, y, z, \tau) d \tau+ \\
& +f_{\Phi_{I}}(x, y, z) \\
& \Phi_{2 V}(x, y, z, t)=\frac{\partial}{\partial x} \int_{0}^{t} D_{\Phi_{V}}(x, y, z, T) \frac{\partial \Phi_{1 V}(x, y, z, \tau)}{\partial x} d \tau+\frac{\partial}{\partial y_{0}^{t}} \int_{0}^{\partial \Phi_{1 V}(x, y, z, \tau)} \frac{\partial}{\partial y} \times \\
& \times D_{\Phi_{V}}(x, y, z, T) d \tau+\frac{\partial}{\partial z} \int_{0}^{t} D_{\Phi_{V}}(x, y, z, T) \frac{\partial \Phi_{1 V}(x, y, z, \tau)}{\partial z} d \tau+\Omega \frac{\partial}{\partial x} \int_{0}^{t} \nabla_{S} \mu(x, y, z, \tau) \times \\
& \times \frac{D_{\Phi_{V} S}}{k T} \int_{0}^{L_{z}}\left[\alpha_{2 \Phi_{V}}+\Phi_{1 V}(x, y, W, \tau)\right] d W d \tau+\Omega \frac{\partial}{\partial y} \int_{0}^{t} \frac{D_{\Phi_{V} S}}{k T} \int_{0}^{L_{z}}\left[\alpha_{2 \Phi_{V}}+\Phi_{1 V}(x, y, W, \tau)\right] d W \times \\
& \times \nabla_{S} \mu(x, y, z, \tau) d \tau+\int_{0}^{t} k_{V, V}(x, y, z, T) V^{2}(x, y, z, \tau) d \tau+\int_{0}^{t} k_{V}(x, y, z, T) V(x, y, z, \tau) d \tau+ \\
& +f_{\Phi_{V}}(x, y, z)
\end{aligned}
$$

Average values of the second-order approximations of required approximations have been calculated by using the following standard relation [26]

$$
\alpha_{2 \rho}=\frac{1}{\Theta L_{x} L_{y} L_{z}} \int_{0}^{\Theta} \int_{0}^{L_{x}} \int_{0}^{L_{y} L_{z}}\left[\rho_{0}(x, y, z, t)-\rho_{1}(x, y, z, t)\right] d z d y d x d t
$$

Substitution of the relations (1e), (3e), (5e) into relation (10) gives us possibility to obtain relations for required average values $\alpha_{2 \rho}$

$$
\begin{aligned}
& \alpha_{2 \mathrm{C}}=0, \alpha_{2 \Phi \mathrm{I}}=0, \alpha_{2 \Phi \mathrm{V}}=0, \quad \sqrt{\frac{\left(b_{3}+E\right)^{2}}{4 b_{4}^{2}}-4\left(F+\frac{\Theta a_{3} F+\Theta^{2} L_{x} L_{y} L_{z} b_{1}}{b_{4}}\right)}-\frac{b_{3}+E}{4 b_{4}}, \\
& \alpha_{2 I}=\frac{C_{V}-\alpha_{2 V}^{2} S_{V V 00}-\alpha_{2 V}\left(2 S_{V V 01}+S_{I V 10}+\Theta L_{x} L_{y} L_{z}\right)-S_{V V 02}-S_{I V 11}}{S_{I V 01}+\alpha_{2 V} S_{I V 00}}
\end{aligned}
$$

where $b_{4}=\frac{1}{\Theta L_{x} L_{y} L_{z}} S_{I V 00}^{2} S_{V V 00}-\frac{1}{\Theta L_{x} L_{y} L_{z}} S_{V V 00}^{2} S_{I I 00}, b_{3}=-\frac{S_{I I 00} S_{V V 00}}{\Theta L_{x} L_{y} L_{z}}\left(2 S_{V V 01}+S_{I V 10}+\right.$ 


$$
\begin{aligned}
& \left.+\Theta L_{x} L_{y} L_{z}\right)+\frac{S_{I V 00} S_{V V 00}}{\Theta L_{x} L_{y} L_{z}}\left(S_{I V 01}+2 S_{I I 10}+S_{I V 01}+\Theta L_{x} L_{y} L_{z}\right)+\frac{S_{I V 00}^{2}}{\Theta L_{x} L_{y} L_{z}}\left(2 S_{V V 01}+S_{I V 10}+\right. \\
& \left.+\Theta L_{x} L_{y} L_{z}\right)-\frac{S_{I V 00}^{2} S_{I V 10}}{\Theta^{3} L_{x}^{3} L_{y}^{3} L_{z}^{3}} \\
& b_{2}=\frac{S_{I I 00} S_{V V 00}}{\Theta L_{x} L_{y} L_{z}}\left(S_{V V 02}+S_{I V 11}+C_{V}\right)-\left(S_{I V 10}-2 S_{V V 01}+\Theta L_{x} L_{y} \times\right. \\
& \left.\times L_{z}\right)^{2}+\frac{S_{I V 01} S_{V V 00}}{\Theta L_{x} L_{y} L_{z}}\left(\Theta L_{x} L_{y} L_{z}+2 S_{I I 10}+S_{I V 01}\right)+\frac{S_{I V 00}}{\Theta L_{x} L_{y} L_{z}}\left(S_{I V 01}+2 S_{I I 10}+2 S_{I V 01}+\Theta L_{x} L_{y} \times\right. \\
& \left.\times L_{z}\right)\left(2 S_{V V 01}+\Theta L_{x} L_{y} L_{z}+S_{I V 10}\right)-\frac{S_{I V 00}^{2}}{\Theta L_{x} L_{y} L_{z}}\left(C_{V}-S_{V V 02}-S_{I V 11}\right)+\frac{C_{I} S_{I V 00}^{2}}{\Theta^{2} L_{x}^{2} L_{y}^{2} L_{z}^{2}}-\frac{2 S_{I V 10}}{\Theta L_{x} L_{y} L_{z}} \times \\
& \times S_{I V 00} S_{I V 01} \\
& b_{1}=S_{I I 00} \frac{S_{I V 11}+S_{V V 02}+C_{V}}{\Theta L_{x} L_{y} L_{z}}\left(2 S_{V V 01}+S_{I V 10}+\Theta L_{x} L_{y} L_{z}\right)+\frac{S_{I V 01}}{\Theta L_{x} L_{y} L_{z}}\left(\Theta L_{x} L_{y} \times\right. \\
& \left.\times L_{z}+2 S_{I I 10}+S_{I V 01}\right)\left(2 S_{V V 01}+S_{I V 10}+\Theta L L_{y} L_{z}\right)-\frac{S_{I V 10} S_{I V 01}^{2}}{\Theta L_{x} L_{y} L_{z}}-\frac{S_{I V 00}}{\Theta L_{x} L_{y} L_{z}}\left(3 S_{I V 01}+2 S_{I I 10}+\right. \\
& \left.+\Theta L_{x} L_{y} L_{z}\right)\left(C_{V}-S_{V V 02}-S_{I V 11}\right)+2 C_{I} S_{I V 00} S_{I V 01} \\
& b_{0}=\frac{S_{I I 00}}{\Theta L_{x} L_{y} L_{z}}\left(S_{I V 00}+S_{V V 02}\right)^{2}-\frac{S_{I V 01}}{L_{x} L_{y} L_{z}} \times \\
& \times \frac{1}{\Theta}\left(\Theta L_{x} L_{y} L_{z}+2 S_{I I 10}+S_{I V 01}\right)\left(C_{V}-S_{V V 02}-S_{I V 11}\right)+2 C_{I} S_{I V 01}^{2}-S_{I V 01} \frac{C_{V}-S_{V V 02}-S_{I V 11}}{\Theta L_{x} L_{y} L_{z}} \times \\
& \times\left(\Theta L_{x} L_{y} L_{z}+2 S_{I I 10}+S_{I V 01}\right), C_{I}=\frac{\alpha_{1 I} \alpha_{1 V}}{\Theta L_{x} L_{y} L_{z}} S_{I V 00}+\frac{\alpha_{1 I}^{2} S_{I I 00}}{\Theta L_{x} L_{y} L_{z}}-\frac{S_{I I 20} S_{I I 20}}{\Theta L_{x} L_{y} L_{z}}-\frac{S_{I V 11}}{\Theta L_{x} L_{y} L_{z}}, \\
& C_{V}=\alpha_{1 I} \alpha_{1 V} S_{I V 00}+\alpha_{1 V}^{2} S_{V V 00}-S_{V V 02}-S_{I V 11}, E=\sqrt{8 y+\Theta^{2} \frac{a_{3}^{2}}{a_{4}^{2}}-4 \Theta \frac{a_{2}}{a_{4}}}, F=\frac{\Theta a_{2}}{6 a_{4}}+ \\
& +\sqrt[3]{\sqrt{r^{2}+s^{3}}-r}-\sqrt[3]{\sqrt{r^{2}+s^{3}}+r}, r=\frac{\Theta^{3} b_{2}}{24 b_{4}^{2}}\left(4 b_{0}-\Theta L_{x} L_{y} L_{z} \frac{b_{1} b_{3}}{b_{4}}\right)-\frac{\Theta^{3} b_{2}^{3}}{54 b_{4}^{3}}-b_{0} \frac{\Theta^{2}}{8 b_{4}^{2}} \times \\
& \times\left(4 \Theta b_{2}-\Theta^{2} \frac{b_{3}^{2}}{b_{4}}\right)-L_{x}^{2} L_{y}^{2} L_{z}^{2} \frac{\Theta^{4} b_{1}^{2}}{8 b_{4}^{2}}, s=\Theta^{2} \frac{4 b_{0} b_{4}-\Theta L_{x} L_{y} L_{z} b_{1} b_{3}}{12 b_{4}^{2}}-\frac{\Theta b_{2}}{18 b_{4}} \text {. }
\end{aligned}
$$

Farther we solve solutions of Eqs.(8), i.e. we determine components of displacement vector. To determine the first-order approximations of the considered components framework method of averaging of function corrections we replace the required functions in the right sides of the equations by their not yet known average values $\alpha_{i}$. The substitution leads to the following result 


$$
\begin{gathered}
\\
\rho(z) \frac{\partial^{2} u_{1 x}(x, y, z, t)}{\partial t^{2}}=-K(z) \beta(z) \frac{\partial T(x, y, z, t)}{\partial x} \quad \rho(z) \frac{\partial^{2} u_{1 y}(x, y, z, t)}{\partial t^{2}}= \\
=-K(z) \beta(z) \frac{\partial T(x, y, z, t)}{\partial y} \quad \rho(z) \frac{\partial^{2} u_{1 z}(x, y, z, t)}{\partial t^{2}}=-K(z) \beta(z) \frac{\partial T(x, y, z, t)}{\partial z}
\end{gathered}
$$

Integration of the left and the right sides of the above relations on time $t$ leads to the following result

$$
\begin{gathered}
u_{1 x}(x, y, z, t)=u_{0 x}+K(z) \frac{\beta(z)}{\rho(z)} \frac{\partial}{\partial x} \int_{0}^{t} \int_{0}^{\vartheta} T(x, y, z, \tau) d \tau d \vartheta- \\
-K(z) \frac{\beta(z)}{\rho(z)} \frac{\partial}{\partial x} \int_{0}^{\infty \vartheta} T(x, y, z, \tau) d \tau d \vartheta \\
u_{1 y}(x, y, z, t)=u_{0 y}+K(z) \frac{\beta(z)}{\rho(z)} \frac{\partial}{\partial y} \int_{0}^{t} \int_{0}^{\vartheta} T(x, y, z, \tau) d \tau d \vartheta- \\
-K(z) \frac{\beta(z)}{\rho(z)} \frac{\partial}{\partial y} \int_{0}^{\infty \vartheta} \int_{0}^{\vartheta} T(x, y, z, \tau) d \tau d \vartheta \\
u_{1 z}(x, y, z, t)=u_{0 z}+K(z) \frac{\beta(z)}{\rho(z)} \frac{\partial}{\partial z} \int_{0}^{t} \int_{0}^{\vartheta} T(x, y, z, \tau) d \tau d \vartheta- \\
-K(z) \frac{\beta(z)}{\rho(z)} \frac{\partial}{\partial z} \int_{0}^{\infty} \int_{0}^{\vartheta} T(x, y, z, \tau) d \tau d \vartheta
\end{gathered}
$$

Approximations with the second and higher orders of components of displacement vector could be determined by using standard replacement of the required components on the following sums $\alpha_{i}+u_{i}(x, y, z, t)$ [26]. The replacement leads to the following result

$$
\begin{aligned}
& \rho(z) \frac{\partial^{2} u_{2 x}(x, y, z, t)}{\partial t^{2}}=\left\{K(z)+\frac{5 E(z)}{6[1+\sigma(z)]}\right\} \frac{\partial^{2} u_{1 x}(x, y, z, t)}{\partial x^{2}}+\left\{K(z)-\frac{E(z)}{3[1+\sigma(z)]\}} \times\right. \\
& \times \frac{\partial^{2} u_{1 y}(x, y, z, t)}{\partial x \partial y}+\frac{E(z)}{2[1+\sigma(z)]}\left[\frac{\partial^{2} u_{1 y}(x, y, z, t)}{\partial y^{2}}+\frac{\partial^{2} u_{1 z}(x, y, z, t)}{\partial z^{2}}\right]-\frac{\partial T(x, y, z, t)}{\partial x} \times \\
& \times K(z) \beta(z)+\left\{K(z)+\frac{E(z)}{3[1+\sigma(z)]}\right\} \frac{\partial^{2} u_{1 z}(x, y, z, t)}{\partial x \partial z} \\
& \left.\rho(z) \frac{\partial^{2} u_{2 y}(x, y, z, t)}{\partial t^{2}}=\frac{E(z)}{2[1+\sigma(z)]\left[\frac{\partial^{2} u_{1 y}(x, y, z, t)}{\partial x^{2}}+\frac{\partial^{2} u_{1 x}(x, y, z, t)}{\partial x \partial y}\right]-\frac{\partial T(x, y, z, t)}{\partial y} \times}\right] \\
& \times K(z) \beta(z)+\frac{\partial}{\partial z}\left\{\frac{E(z)}{\left.2[1+\sigma(z)]\left[\frac{\partial u_{1 y}(x, y, z, t)}{\partial z}+\frac{\partial u_{1 z}(x, y, z, t)}{\partial y}\right]\right\}+\frac{\partial^{2} u_{1 y}(x, y, z, t)}{\partial y^{2}} \times}\right.
\end{aligned}
$$




$$
\begin{aligned}
& \times\left\{\frac{5 E(z)}{12[1+\sigma(z)]}+K(z)\right\}+\left\{K(z)-\frac{E(z)}{6[1+\sigma(z)]}\right\} \frac{\partial^{2} u_{1 y}(x, y, z, t)}{\partial y \partial z}+K(z) \frac{\partial^{2} u_{1 y}(x, y, z, t)}{\partial x \partial y} \\
& \rho(z) \frac{\partial^{2} u_{2 z}(x, y, z, t)}{\partial t^{2}}=\frac{E(z)}{2[1+\sigma(z)]}\left[\frac{\partial^{2} u_{1 z}(x, y, z, t)}{\partial x^{2}}+\frac{\partial^{2} u_{1 z}(x, y, z, t)}{\partial y^{2}}+\frac{\partial^{2} u_{1 x}(x, y, z, t)}{\partial x \partial z}+\right. \\
& \left.+\frac{\partial^{2} u_{1 y}(x, y, z, t)}{\partial y \partial z}\right]+\frac{\partial}{\partial z}\left\{K(z)\left[\frac{\partial u_{1 x}(x, y, z, t)}{\partial x}+\frac{\partial u_{1 y}(x, y, z, t)}{\partial y}+\frac{\partial u_{1 x}(x, y, z, t)}{\partial z}\right]\right\}+ \\
& +\frac{E(z)}{6[1+\sigma(z)]} \frac{\partial}{\partial z}\left[6 \frac{\partial u_{1 z}(x, y, z, t)}{\partial z}-\frac{\partial u_{1 x}(x, y, z, t)}{\partial x}-\frac{\partial u_{1 y}(x, y, z, t)}{\partial y}-\frac{\partial u_{1 z}(x, y, z, t)}{\partial z}\right]- \\
& \left.\left.-\frac{\partial u_{1 x}(x, y, z, t)}{\partial x}-\frac{\partial u_{1 y}(x, y, z, t)}{\partial y}-\frac{\partial u_{1 z}(x, y, z, t)}{\partial z}\right]\right\} \frac{E(z)}{1+\sigma(z)}-K(z) \beta(z) \frac{\partial T(x, y, z, t)}{\partial z}
\end{aligned}
$$

Integration of the left and right sides of the above relations on time $t$ leads to the following result

$$
\begin{aligned}
& u_{2 x}(x, y, z, t)=\frac{1}{\rho(z)}\left\{K(z)+\frac{5 E(z)}{6[1+\sigma(z)]}\right\} \frac{\partial^{2}}{\partial x^{2}} \int_{0}^{t \vartheta} \int_{0}^{\vartheta} u_{1 x}(x, y, z, \tau) d \tau d \vartheta+\frac{1}{\rho(z)}\{K(z)- \\
& \left.-\frac{E(z)}{3[1+\sigma(z)]}\right\} \frac{\partial^{2}}{\partial x \partial y} \int_{0}^{t} \int_{0}^{\vartheta} u_{1 y}(x, y, z, \tau) d \tau d \vartheta+\frac{E(z)}{2 \rho(z)}\left[\frac{\partial^{2}}{\partial y^{2}} \int_{0}^{t} \int_{0}^{\vartheta} u_{1 y}(x, y, z, \tau) d \tau d \vartheta+\right. \\
& \left.+\frac{\partial^{2}}{\partial z^{2}} \int_{0}^{t} \int_{0}^{\vartheta} u_{1 z}(x, y, z, \tau) d \tau d \vartheta\right] \frac{1}{1+\sigma(z)}+\frac{1}{\rho(z)} \frac{\partial^{2}}{\partial x \partial z} \int_{0}^{t} \int_{0}^{\vartheta} u_{1 z}(x, y, z, \tau) d \tau d \vartheta\{K(z)+ \\
& \left.+\frac{E(z)}{3[1+\sigma(z)]}\right\}-K(z) \frac{\beta(z)}{\rho(z)} \frac{\partial}{\partial x} \int_{0}^{t \vartheta} \int_{0}^{\vartheta} T(x, y, z, \tau) d \tau d \vartheta-\frac{\partial^{2}}{\partial x^{2}} \int_{0}^{\infty \vartheta} \int_{0}^{\vartheta} u_{1 x}(x, y, z, \tau) d \tau d \vartheta \times \\
& \times \frac{1}{\rho(z)}\left\{K(z)+\frac{5 E(z)}{6[1+\sigma(z)]}\right\}-\left\{K(z)-\frac{E(z)}{3[1+\sigma(z)]}\right\} \frac{\partial^{2}}{\partial x \partial y} \int_{0}^{\infty \vartheta} \int_{0}^{\vartheta} u_{1 y}(x, y, z, \tau) d \tau d \vartheta \times \\
& \times \frac{1}{\rho(z)}-\frac{E(z)}{2 \rho(z)[1+\sigma(z)]}\left[\frac{\partial^{2}}{\partial y^{2}} \int_{0}^{\infty \vartheta} \int_{0}^{\vartheta} u_{1 y}(x, y, z, \tau) d \tau d \vartheta+\frac{\partial^{2}}{\partial z^{2}} \int_{0}^{\infty \vartheta} \int_{0}^{\vartheta} u_{1 z}(x, y, z, \tau) d \tau d \vartheta\right]- \\
& -\frac{1}{\rho(z)}\left\{K(z)+\frac{E(z)}{3[1+\sigma(z)]}\right\} \frac{\partial^{2}}{\partial x \partial z} \int_{0}^{\infty} \int_{0}^{\vartheta} u_{1 z}(x, y, z, \tau) d \tau d \vartheta+u_{0 x}+K(z) \frac{\beta(z)}{\rho(z)} \times \\
& \times \frac{\partial}{\partial x} \int_{0}^{\infty} \int_{0}^{\vartheta} T(x, y, z, \tau) d \tau d \vartheta \\
& u_{2 y}(x, y, z, t)=\frac{E(z)}{2 \rho(z)}\left[\frac{\partial^{2}}{\partial x^{2}} \int_{0}^{t \vartheta} \int_{0} u_{1 x}(x, y, z, \tau) d \tau d \vartheta+\frac{\partial^{2}}{\partial x \partial y} \int_{0}^{t} \int_{0}^{\vartheta} u_{1 x}(x, y, z, \tau) d \tau d \vartheta\right] \times
\end{aligned}
$$


$\times \frac{1}{1+\sigma(z)}+\frac{K(z)}{\rho(z)} \frac{\partial^{2}}{\partial x \partial y} \int_{0}^{t} \int_{0}^{\vartheta} u_{1 y}(x, y, z, \tau) d \tau d \vartheta+\frac{1}{\rho(z)}\left\{\frac{5 E(z)}{12[1+\sigma(z)]}+K(z)\right\} \times$ $\times \frac{\partial^{2}}{\partial y^{2}} \int_{0}^{t} \int_{0}^{\vartheta} u_{1 x}(x, y, z, \tau) d \tau d \vartheta+\frac{1}{2 \rho(z)} \frac{\partial}{\partial z}\left\{\frac{E(z)}{1+\sigma(z)}\left[\frac{\partial}{\partial z} \int_{0}^{t} \int_{0}^{\vartheta} u_{1 y}(x, y, z, \tau) d \tau d \vartheta+\right.\right.$ $\left.\left.+\frac{\partial}{\partial y} \int_{0}^{t \vartheta} \int_{0}^{\vartheta} u_{1 z}(x, y, z, \tau) d \tau d \vartheta\right]\right\}-K(z) \frac{\beta(z)}{\rho(z)} \int_{0}^{t} \int_{0}^{\vartheta} T(x, y, z, \tau) d \tau d \vartheta-\left\{\frac{E(z)}{6[1+\sigma(z)]}-\right.$ $-K(z)\} \frac{1}{\rho(z)} \frac{\partial^{2}}{\partial y \partial z} \int_{0}^{t} \int_{0}^{\vartheta} u_{1 y}(x, y, z, \tau) d \tau d \vartheta-\frac{E(z)}{2 \rho(z)}\left[\frac{\partial^{2}}{\partial x^{2}} \iint_{0}^{\infty \vartheta} \int_{0}^{\vartheta} u_{1 x}(x, y, z, \tau) d \tau d \vartheta+\right.$ $\left.+\frac{\partial^{2}}{\partial x \partial y} \int_{0}^{\infty \vartheta} \int_{0}^{\vartheta} u_{1 x}(x, y, z, \tau) d \tau d \vartheta\right] \frac{1}{1+\sigma(z)}-K(z) \frac{\beta(z)}{\rho(z)} \int_{0}^{\infty \vartheta} \int_{0}^{\vartheta} T(x, y, z, \tau) d \tau d \vartheta-\frac{K(z)}{\rho(z)} \times$ $\times \frac{\partial^{2}}{\partial x \partial y} \int_{0}^{\infty \vartheta} \int_{0}^{\vartheta} u_{1 y}(x, y, z, \tau) d \tau d \vartheta-\frac{1}{\rho(z)} \frac{\partial^{2}}{\partial y^{2}} \int_{0}^{\infty} \int_{0}^{\vartheta} u_{1 x}(x, y, z, \tau) d \tau d \vartheta\left\{\frac{5 E(z)}{12[1+\sigma(z)]}+\right.$ $+K(z)\}-\frac{\partial}{\partial z}\left\{\frac{E(z)}{1+\sigma(z)}\left[\frac{\partial}{\partial z} \int_{0}^{\infty \vartheta} \int_{0}^{\vartheta} u_{1 y}(x, y, z, \tau) d \tau d \vartheta+\frac{\partial}{\partial y} \int_{0}^{\infty \vartheta} \int_{0}^{\vartheta} u_{1 z}(x, y, z, \tau) d \tau d \vartheta\right] \times x\right.$ $\times \frac{1}{2 \rho(z)}-\frac{1}{\rho(z)}\left\{K(z)-\frac{E(z)}{6[1+\sigma(z)]}\right\} \frac{\partial^{2}}{\partial y \partial z} \int_{0}^{\infty} \int_{0}^{\vartheta} u_{1 y}(x, y, z, \tau) d \tau d \vartheta+u_{0 y}$ $u_{z}(x, y, z, t)=\frac{E(z)}{2[1+\sigma(z)]}\left[\frac{\partial^{2}}{\partial x^{2}} \int_{0}^{\infty \vartheta} \int_{0}^{\vartheta} u_{1 z}(x, y, z, \tau) d \tau d \vartheta+\frac{\partial^{2}}{\partial y^{2}} \int_{0}^{\infty \vartheta} \int_{0}^{\vartheta} u_{1 z}(x, y, z, \tau) d \tau d \vartheta+\right.$ $\left.+\frac{\partial^{2}}{\partial x \partial z} \int_{0}^{\infty \vartheta} \int_{0}^{\vartheta} u_{1 x}(x, y, z, \tau) d \tau d \vartheta+\frac{\partial^{2}}{\partial y \partial z} \int_{0}^{\infty \vartheta} \int_{0}^{\vartheta} u_{1 y}(x, y, z, \tau) d \tau d \vartheta\right] \frac{1}{\rho(z)}+\frac{1}{\rho(z)} \times$ $\times \frac{\partial}{\partial z}\left\{K(z)\left[\frac{\partial}{\partial x} \int_{0}^{\infty \vartheta} \int_{0}^{\vartheta} u_{1 x}(x, y, z, \tau) d \tau d \vartheta+\frac{\partial}{\partial y} \int_{0}^{\infty \vartheta} \int_{0}^{\vartheta} u_{1 x}(x, y, z, \tau) d \tau d \vartheta+\right.\right.$ $\left.\left.+\frac{\partial}{\partial z} \int_{0}^{\infty} \int_{0}^{\vartheta} u_{1 x}(x, y, z, \tau) d \tau d \vartheta\right]\right\}+\frac{1}{6 \rho(z)} \frac{\partial}{\partial z}\left\{\frac{E(z)}{1+\sigma(z)}\left[6 \frac{\partial}{\partial z} \int_{0}^{\infty \vartheta} \int_{0}^{\vartheta} u_{1 z}(x, y, z, \tau) d \tau d \vartheta-\right.\right.$ $\left.\left.-\frac{\partial}{\partial x} \int_{0}^{\infty \vartheta} \int_{0}^{\vartheta} u_{1 x}(x, y, z, \tau) d \tau d \vartheta-\frac{\partial}{\partial y} \int_{0}^{\infty \vartheta} \int_{0}^{\vartheta} u_{1 y}(x, y, z, \tau) d \tau d \vartheta-\frac{\partial}{\partial z} \int_{0}^{\infty \vartheta} \int_{0}^{\vartheta} u_{1 z}(x, y, z, \tau) d \tau d \vartheta\right]\right\}-$ $-K(z) \frac{\beta(z)}{\rho(z)} \frac{\partial}{\partial z} \int_{0}^{\infty \vartheta} \int_{0}^{\vartheta} T(x, y, z, \tau) d \tau d \vartheta+u_{0 z}$ 
In this paper we calculate concentration of dopant, concentrations of radiation defects and components of displacement vector by using the second-order approximation framework method of averaging of function corrections. This approximation is usually enough good approximation to make qualitative analysis and to obtain some quantitative results. All obtained results have been checked by comparison with results of numerical simulations.

\section{Discussion}

In this section based on recently obtained approximations we analyzed redistributions of dopant and radiation defects during annealing and under influence of mismatch-induced stress. As the dopant we consider Ge. As a doped heterostructure we consider SOI structure.
Parameters of technological process are the same excluding annealing time. The annealing time was optimized to increase sharpness of $p$ - $n$-junctions, to decrease dimensions of bipolar transistors and to increase density of these transistors framework integrated circuits. In this situation, recently we had a possibility to compare calculated results with experimental one [25]. Typical distributions of concentrations of dopant in heterostructures are presented on Figs. 2 and 3 for diffusion and ion types of doping, respectively. These distributions have been calculated for the case, when value of dopant diffusion coefficient in the epitaxial layer is larger, than in the substrate. The figures show that inhomogeneity of heterostructure gives us possibility to increase compactness of transistors. At the same time one can find increasing homogeneity of dopant distribution in doped part of epitaxial layer. In-creasing of compactness of transistors gives us possibility to increase their density.

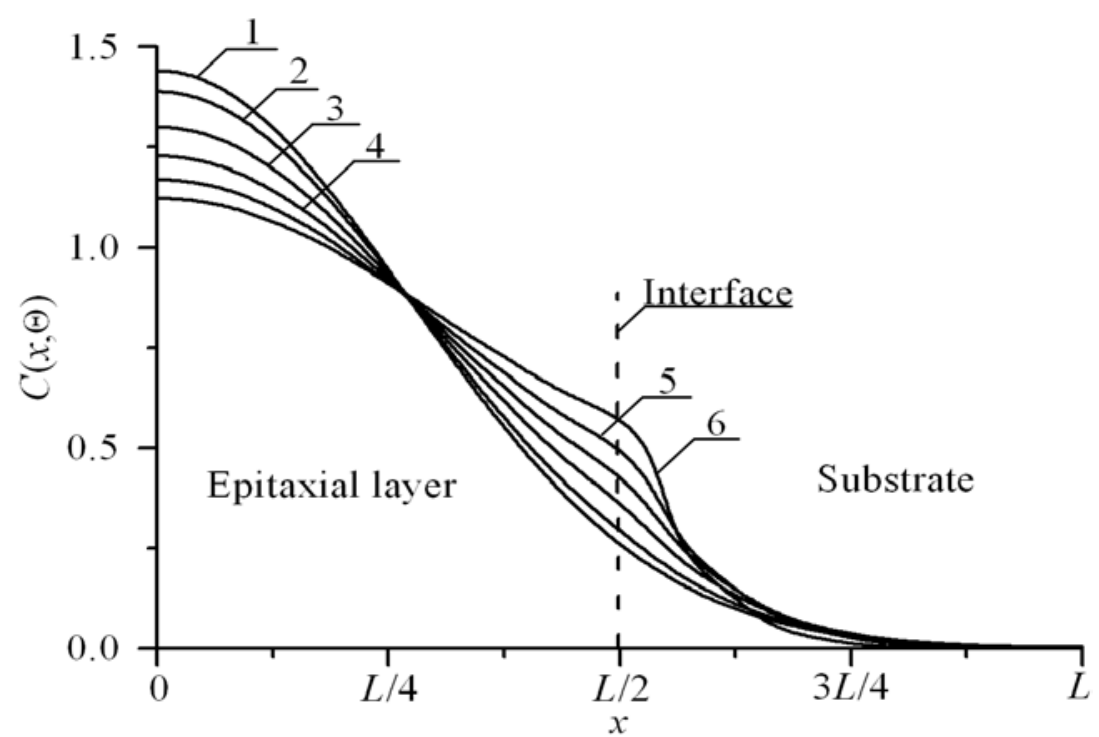

Figure 2. Distributions of concentration of infused dopant in heterostructure from Fig. 1 in direction, which is perpendicular to interface between epitaxial layer substrate. Increasing of number of curve corresponds to increasing of difference between values of dopant diffusion coefficient in layers of heterostructure under condition, when value of dopant diffusion coefficient in epitaxial layer is larger, than value of dopant diffusion coefficient in substrate 


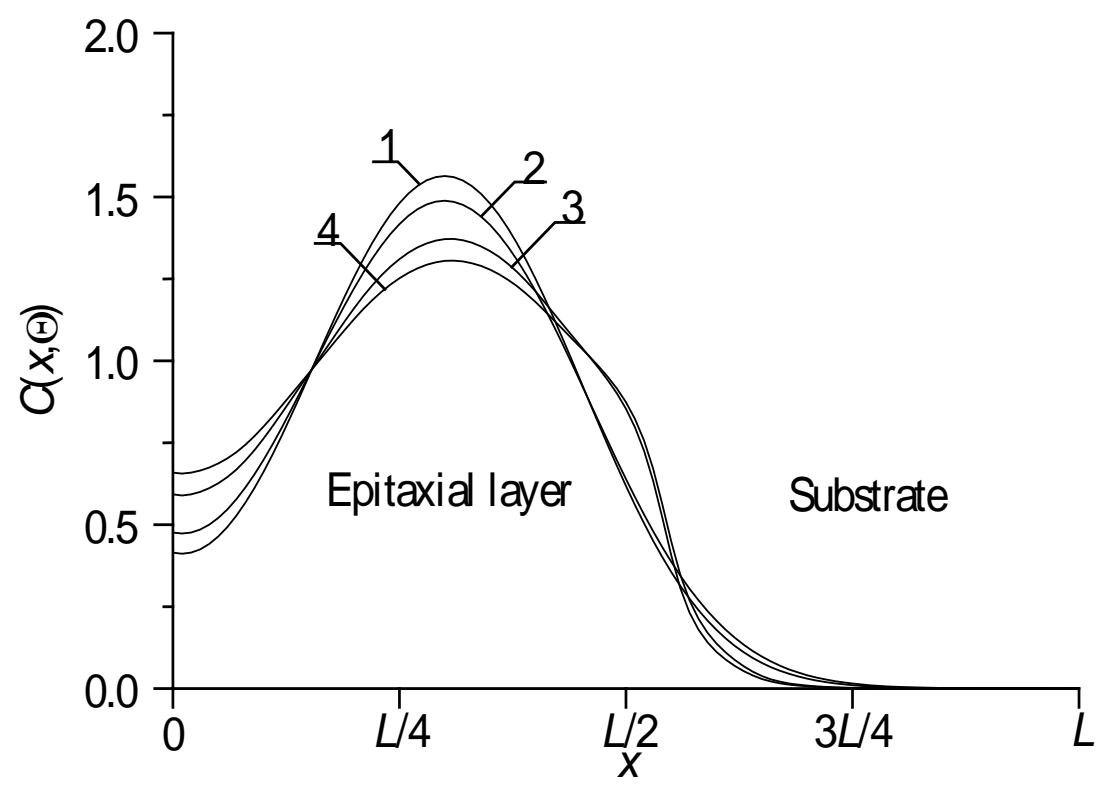

Figure 3. Distributions of concentration of implanted dopant in heterostructure from Fig. 1 in direction, which is perpendicular to interface between epitaxial layer substrate. Curves 1 and 3 corresponds to annealing time $\Theta=0.0048\left(L_{x}{ }^{2}+L_{y}{ }^{2}+L_{z}{ }^{2}\right) / D_{0}$. Curves 2 and 4 corresponds to annealing time $\Theta=$ $0.0057\left(L_{x}{ }^{2}+L_{y}{ }^{2}+L_{z}{ }^{2}\right) / D_{0}$. Curves 1 and 2 correspond to homogenous sample. Curves 3 and 4 corresponds to heterostructure under condition, when value of dopant diffusion coefficient in epitaxial layer is larger, than value of dopant diffusion coefficient in substrate

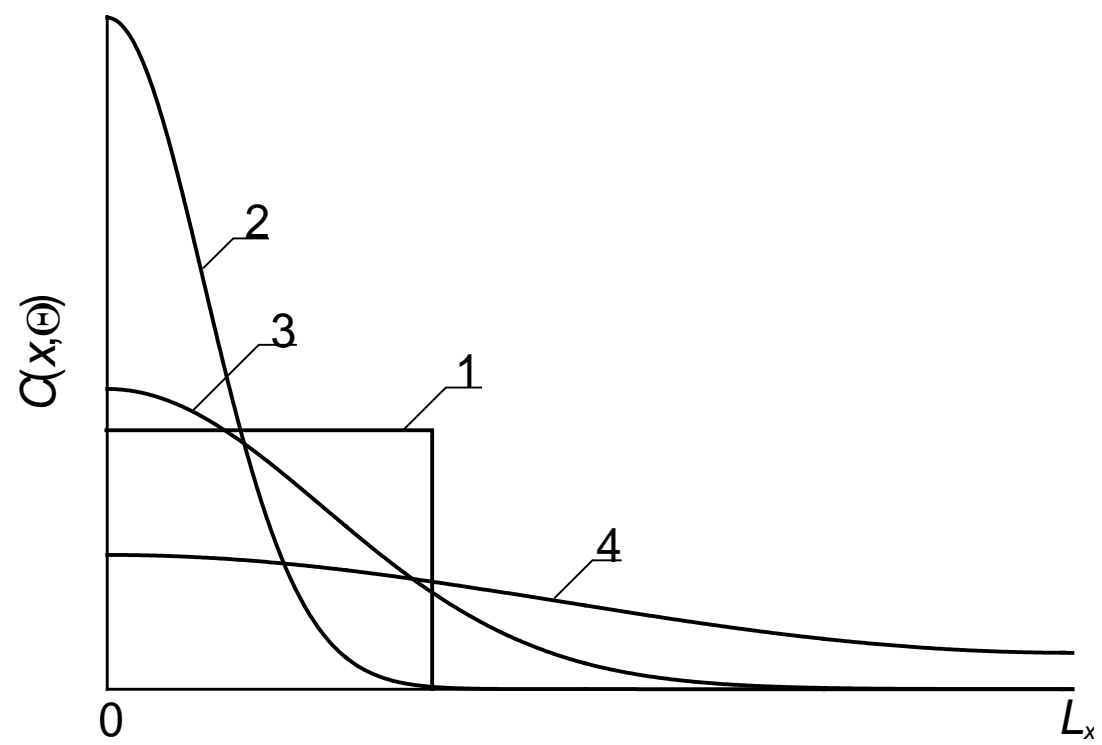

Figure 4. Spatial distributions of dopant in heterostructure after dopant infusion. Curve 1 is idealized distribution of dopant. Curves 2-4 are real distributions of dopant for different values of annealing time. Increasing of number of curve corresponds to increasing of annealing time 


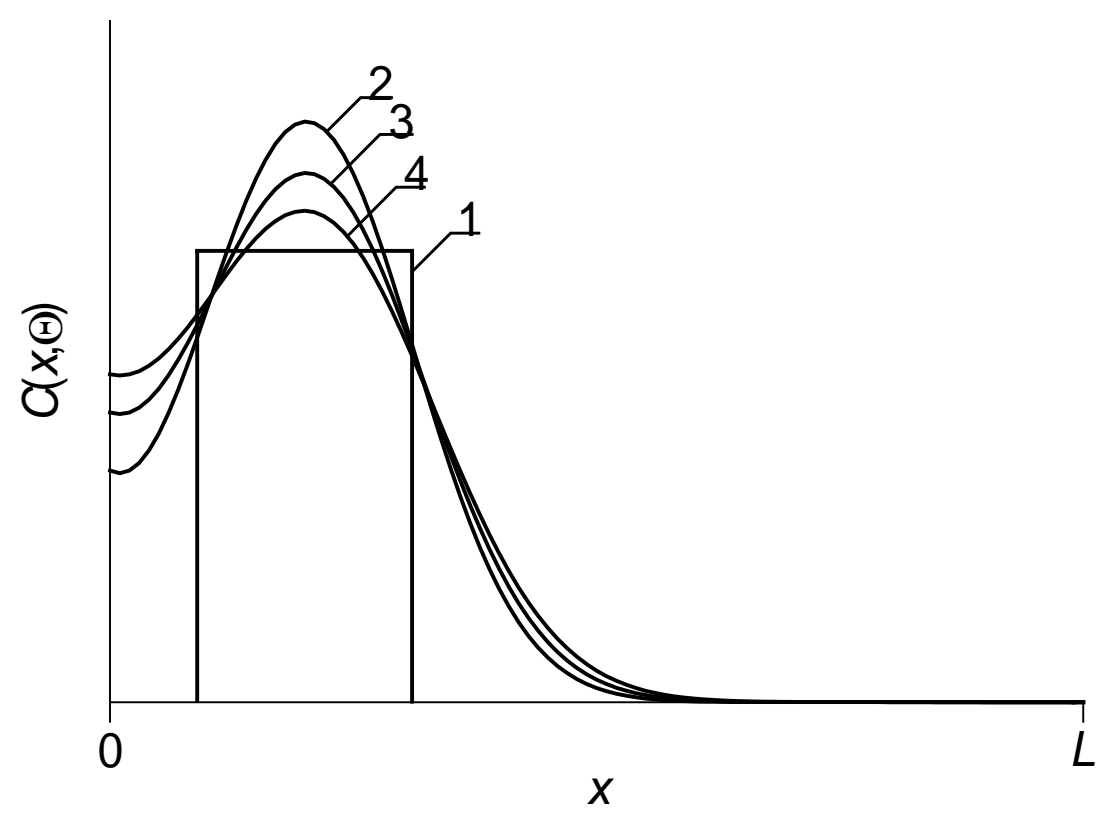

Figure 5. Spatial distributions of dopant in heterostructure after ion implantation. Curve 1 is idealized distribution of dopant. Curves 2-4 are real distributions of dopant for different values of annealing time. Increasing of number of curve corresponds to increasing of annealing time

The second effect leads to decreasing of local heating of materials during functioning of transistors or decreasing of their dimensions for fixed maximal value of local overheat. However, framework this approach of manufacturing of bipolar transistor it is necessary to optimize annealing of dopant and/or radiation defects. Reason of this optimization is following. If annealing time is small, the dopant did not achieve any interfaces between materials of heterostructure. In this situation, one cannot find any modifications of distribution of concentration of dopant. If annealing time is large, distribution of concentration of dopant became too homogenous. We optimize annealing time by using recently introduces approach [15, 26-33]. Framework of this approach, we approximate real distribution of concentration of dopant by step-wise function (see Figs. 4 and 5). Farther we determine optimal values of annealing time by minimization of the following mean-squared error

$$
U=\frac{1}{L_{x} L_{y} L_{z}} \int_{0}^{L_{x}} \int_{0}^{L_{y} L_{z}} \int_{0}[C(x, y, z, \Theta)-\psi(x, y, z)] d z d y d x,
$$

where $\psi(x, y, z)$ is the considered approximation function. Dependences of optimal values of annealing time on parameters are presented on Figs. 6 and 7 for diffusion and ion types of doping, respectively. It should be noted, that it is necessary to anneal radiation defects after ion implantation. One could find spreading of concentration of distribution of dopant during this annealing. In the ideal case distribution of dopant achieves appropriate interfaces between materials of heterostructure during annealing of radiation defects. If dopant did not achieve any interfaces during annealing of radiation defects, it is practicably to use additional annealing of dopant. In this situation optimal value of additional annealing time of implanted dopant is smaller, than annealing time of infused dopant. 


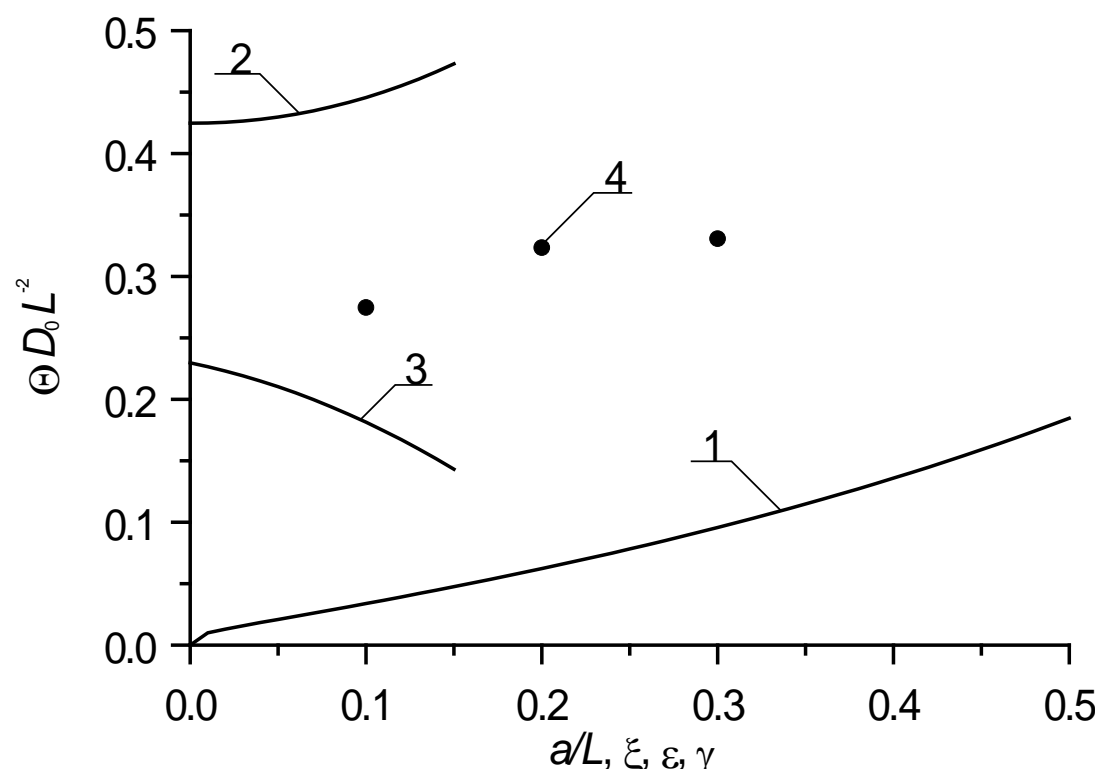

Figure 6. Dependences of dimensionless optimal annealing time doping by diffusion, which have been obtained by minimization of mean-squared error (15), on several parameters. Curve 1 is the dependence of dimensionless optimal annealing time on the relation $a / L$ and $\xi=\gamma=0$ for equal to each other values of dopant diffusion coefficient in all parts of heterostructure. Curve 2 is the dependence of dimensionless optimal annealing time on value of parameter $\varepsilon$ for $a / L=1 / 2$ and $\xi=\gamma=0$. Curve 3 is the dependence of dimensionless optimal annealing time on value of parameter $\xi$ for $a / L=1 / 2$ and $\varepsilon$ $=\gamma=0$. Curve 4 is the dependence of dimensionless optimal annealing time on value of parameter $\gamma$ for $a / L=1 / 2$ and $\varepsilon=\xi=0$

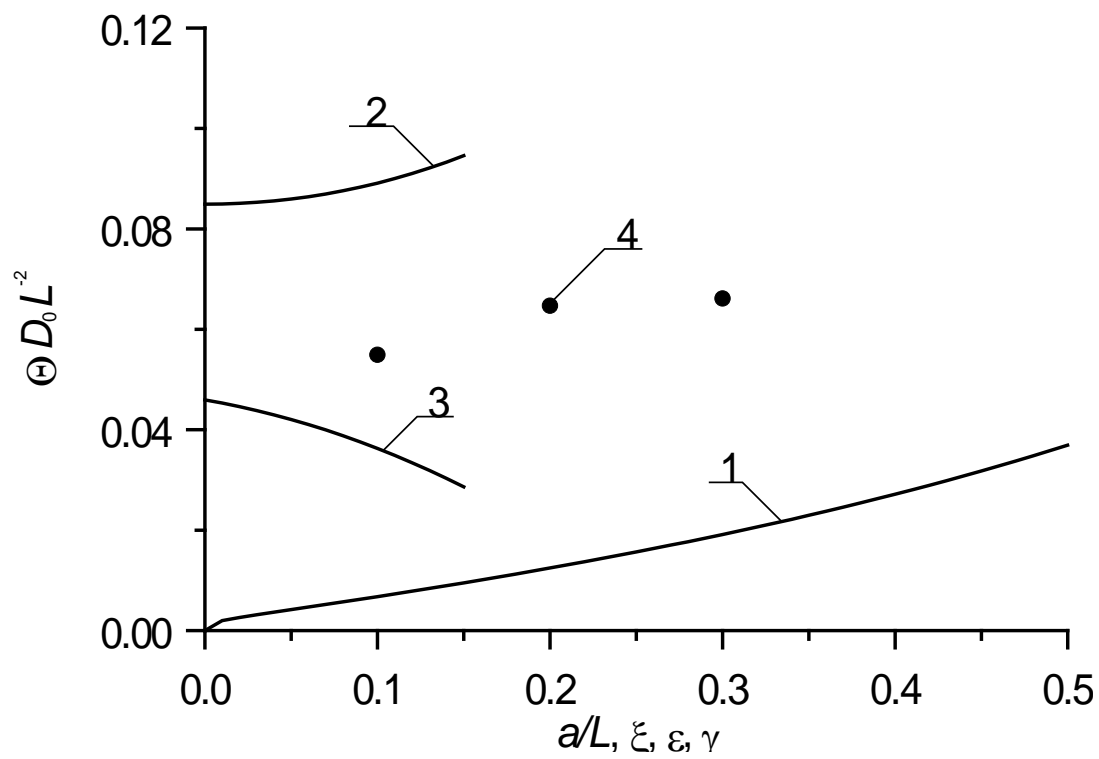

Figure 7. Dependences of dimensionless optimal annealing time for doping by ion implantation, which have been obtained by minimization of mean-squared error, on several parameters. Curve 1 is the dependence of dimensionless optimal annealing time on the relation $a / L$ and $\xi=\gamma=0$ for equal to each other values of dopant diffusion coefficient in all parts of heterostructure. Curve 2 is the dependence of dimensionless optimal annealing time on value of parameter $\varepsilon$ for $a / L=1 / 2$ and $\xi=\gamma=0$. Curve 3 is the dependence of dimensionless optimal annealing time on value of parameter $\xi$ for $a / L=1 / 2$ and $\varepsilon=\gamma=0$. Curve 4 is the dependence of dimensionless optimal annealing time on value of parameter $\gamma$ for $a / L=1 / 2$ and $\varepsilon=\xi=0$ 


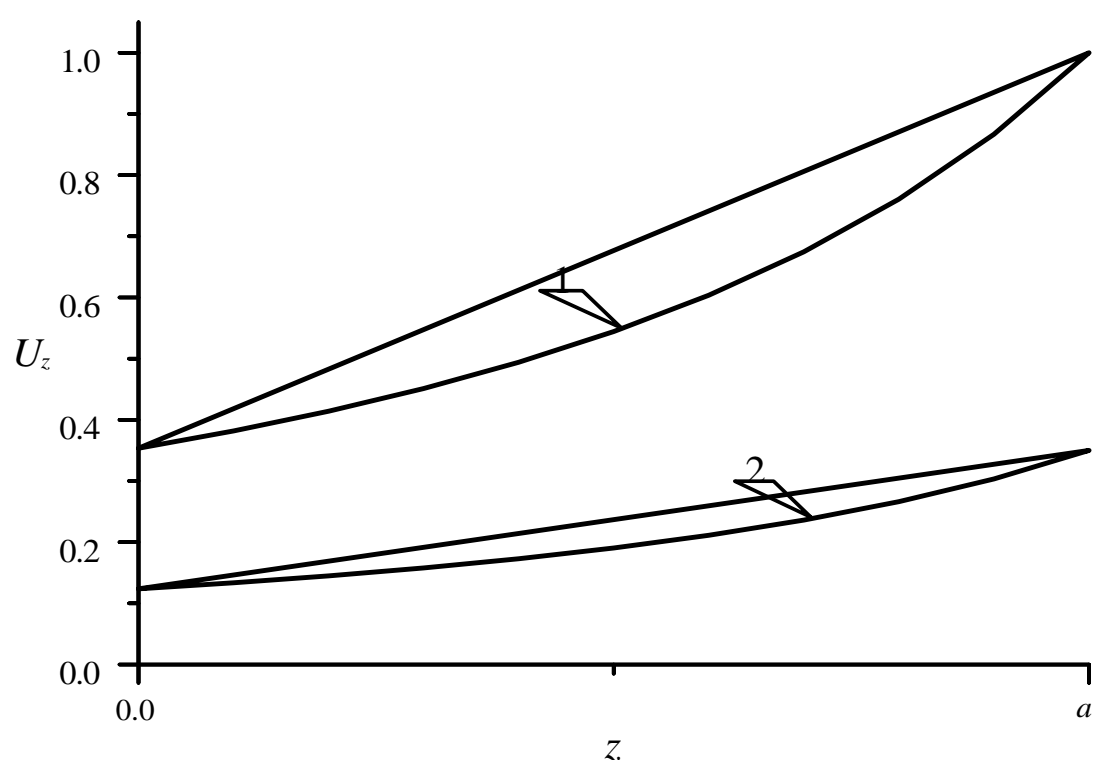

Figure 8. Normalized dependences of component $u_{z}$ of displacement vector on coordinate $z$. Curve 1 corresponds to not processed (without radiation processing) epitaxial layers. Curve 2 corresponds to processed epitaxial layers (after radiation processing).

Now we analyzed influence of relaxation of mechanical stress on distribution of dopant in doped areas of heterostructure. Under following condition $\varepsilon_{0}<0$ one can find compression of distribution of concentration of dopant near interface between materials of heterostructure. Contrary (at $\varepsilon_{0}>0$ ) one can find spreading of distribution of concentration of dopant in this area. This changing of distribution of concentration of dopant could be at least partially compensated by using laser annealing [30]. This type of annealing gives us possibility to accelerate diffusion of dopant and other processes in annealed area due to inhomogenous distribution of temperature and Arrhenius law. Accounting relaxation of mismatch-induced stress in heterostructure could leads to changing of optimal values of annealing time. Mismatch-induced stress could be used to increase density of elements of integrated circuits. On the other hand could leads to generation dislocations of the discrepancy. Fig. 8 shows distributions of component of displacement vector, which is perpendicular to interface between layers of heterostructure.

\section{Conclusions}

In this paper we model redistribution of infused and implanted dopants with account relaxation mismatch-induced stress during manufacturing a bipolar heterotransistors framework a circuit of voltage divider biasing common emitter amplifier. We formulate recommendations for optimization of annealing to decrease dimensions of transistors and to increase their density. We formulate recommendations to decrease mismatch-induced stress. Analytical approach to model diffusion and ion types of doping with account concurrent changing of parameters in space and time has been introduced. At the same time the approach gives us possibility to take into account nonlinearity of considered processes.

\section{REFERENCES}

[1] V.I. Lachin, and N.S. Savelov, Electronics, Rostov-on-Don: Phoenix (2001).

[2] A. Polishscuk, Modern Electronics, Issue 12, 8 (2004).

[3] G. Volovich, Modern Electronics,. Issue 2, 10 (2006).

[4] A. Kerentsev, and V. Lanin, Power Electronics, Issue 1, 34 (2008).

[5] A.O. Ageev, A.E. Belyaev, N.S. Boltovets, V.N. Ivanov, R.V. Konakova, Ya.Ya. Kudrik, P.M. Litvin, V.V. Milenin, and A.V. Sachenko, Semiconductors, 43, 897 (2009).

[6] Jung-Hui Tsai, Shao-Yen Chiu, Wen-Shiung Lour, and Der-Feng Guo, Semiconductors, 43, 971 (2009).

[7] O.V. Alexandrov, A.O. Zakhar'in, N.A. Sobolev, E.I. Shek, M.M. Makoviychuk, and E.O. Parshin, Semiconductors, 32, 1029 (1998).

[8] I.B. Ermolovich, V.V. Milenin, R.A. Red'ko, and S.M. Red'ko, Semiconductors, 43, 1016 (2009).

[9] P. Sinsermsuksakul, K. Hartman, S.B. Kim, J. Heo, L. Sun, H.H. Park, R. Chakraborty, T. Buonassisi, and R.G. Gordon, Appl. Phys. Lett., 102, 053901 (2013).

[10] J.G. Reynolds, C.L. Reynolds, Jr.A. Mohanta, J.F. Muth, J.E. Rowe, H.O. Everitt, and D.E. Aspnes, Appl. Phys. Lett., 102, 152114 (2013).

[11] N.I. Volokobinskaya, I.N. Komarov, T.V. Matyukhina, V.I. Reshetnikov, A.A. Rush, I.V. Falina, and A.S. Yastrebov, 
Semiconductors, 35, 1013 (2001).

[12] E.L. Pankratov, and E.A. Bulaeva, Reviews in Theoretical Science, 1, 58 (2013).

[13] X. Chen, J. Xue, Sh. Xie, W. Huang, P. Wang, K. Gong, and L. Zhong, Circuits and Systems, 8, 247 (2017).

[14] S.A. Kukushkin, A.V. Osipov, and A.I. Romanychev, Physics of the Solid State, 58, 1448 (2016).

[15] E.M. Trukhanov, A.V. Kolesnikov, and I. D. Loshkarev, Russian Microelectronics, 44, 552 (2015).

[16] E.L. Pankratov, and E.A. Bulaeva, Reviews in Theoretical Science, 3, 365 (2015).

[17] K.K. Ong, K.L. Pey, P.S. Lee, A.T.S. Wee, X.C. Wang, and Y.F. Chong, Appl. Phys. Lett., 89, 172111 (2006).

[18] H.T. Wang, L.S. Tan, and E.F. Chor, J. Appl. Phys., 98, 094901 (2006).

[19] Yu.V. Bykov, A.G. Yeremeev, N.A. Zharova, I.V. Plotnikov, K.I. Rybakov, M.N. Drozdov, Yu.N. Drozdov, and V.D. Skupov, Radiophysics and Quantum Electronics, 43, 836 (2003).

[20] Y.W. Zhang, and A.F. Bower, Journal of the Mechanics and Physics of Solids, 47, 2273 (1999).

[21] L.D. Landau, and E.M. Lefshits, Theoretical physics, 7 (Theory of elasticity), Physmatlit, Moscow (2001).

[22] Z.Yu. Gotra, Technology of microelectronic devices, Radio and communication, Moscow (1991).

[23] P.M. Fahey, P.B. Griffin, and J.D. Plummer. Rev. Mod. Phys., 61, 289 (1989).

[24] V.L. Vinetskiy, and G.A. Kholodar', Radiative physics of semiconductors, "Naukova Dumka", Kiev (1979).

[25] E.L. Pankratov, and E.A. Bulaeva, Int. J. Micro-Nano Scale Transp., 4, 17 (2014).

[26] E.L. Pankratov, O.P. Gus'kova, M.N. Drozdov, N.D. Abrosimova, and V.M. Vorotyntsev, Semiconductors, 48, 612 (2014).

[27] Yu.D. Sokolov, Applied Mechanics, 1, 23 (1955).

[28] E.L. Pankratov, Russian Microelectronics, 36, 33 (2007).

[29] E.L. Pankratov, Int. J. Nanoscience, 7, 187 (2008).

[30] E.L. Pankratov, and E.A. Bulaeva, Int. J. Micro-Nano Scale Transp., 3, 119 (2012).

[31] E.L. Pankratov, Nano, 6, 31 (2011).

[32] E.L. Pankratov, and E.A. Bulaeva, J. Comp. Theor. Nanoscience, 10, 888 (2013).

[33] E.L. Pankratov, and E.A. Bulaeva, J. Nanoeng. Nanoman, 6, 313 (2016). 